\title{
A Time Domain Model for the Study of High Frequency 3D Wheelset-track Interaction with non-Hertzian contact
}

\begin{abstract}
A novel numerical model for train-track interaction is proposed in this paper to deal with wheel-rail interface dynamics in high frequency range. The complete model consists a 3D rotating flexible wheelset model, a 3D track model considering the discrete support of the rail and a non-linear, non-Hertzian model of wheel/rail contact. The wheelset and the track models are both defined using an 'Arbitrary LagrangianEulerian' Finite Element approach in combination with modal synthesis. This allows an efficient treatment of the problem, compared to a classical Finite Element approach. The proposed model is suitable to represent train-track interaction effects in a frequency range up to $7 \mathrm{kHz}$ thanks to the detailed description of wheelset and rail deformability. Wheel/rail contact forces and rail vibration under excitation produced by different types of railhead irregularity are investigated in the paper, assessing the effect of different models of wheelset and track flexibility. The results obtained show that the outputs of the model mostly relevant to the investigation of rail corrugation and rolling noise, i.e. wheel-rail contact forces and rail vibration, are highly sensitive to the wheelset and track model adopted.
\end{abstract}

\section{Introduction}

The demand of higher running speed for long-distance passenger trains led to increasing dynamic problems of interaction between vehicle and track, such as high levels of noise [1] and vibration [2], wheel-rail forces and axle stress fluctuations [3], growth of outof-roundness of wheels [4] as well as corrugation of track [5]. Simulation tools for traintrack interaction system dynamics, including the vehicle model, the track model as well as the contact model, have therefore been extensively studied over the last 45 years.

Train-track interaction dynamics can be solved both in frequency domain and in time domain. Rolling noise [1] and vehicle-track coupled vibration response caused by the roughness of the wheel and rail running surfaces [2] have often been investigated using linear frequency-domain wheel/rail interaction models, the dynamic response characteristics at wheel-rail contact being derived by combining the wheelset and track frequency response functions with the assumed rail roughness [6]. However, frequency domain methods cannot account for nonlinear effects in wheel-rail contact, provide an accurate prediction of wheel-rail contact conditions and consider the effect of local 
wheel/rail defects. To account for these nonlinear effects, train-track interaction models in time domain are required.

Recent research on train-track interaction in time domain has been devoted to developing wheelset/track models based on the Finite Element (FE) method and a more realistic contact model. While the vehicle has been modelled as multi-body systems with the track as Euler-Bernoulli or Timoshenko beams in last decades [7], recent trends of vehicles modelling in high frequency considering flexible wheelset and track modelling in general FE method. In order to simplify the large dimension FE integration, the 'Arbitrary Lagrangian-Eulerian' (ALE) approach has been widely used for the flexible wheelset and track model although the term ALE may have not been adopted. To investigate wheelset dynamics, Baeza et al. [3, 8, 9] and Kaiser and coauthors [10-13] proposed the use of the ALE approach introducing an intermediate reference frame that moves with the rotating wheelset, so that a fixed longitudinal position of the contact point can be considered. Baeza et al. [8] evaluated the gradients of the shape function by making a linear transformation of Brown and Shabana's formulation $[14,15]$ of kinematics of flexible rotating structure in a Lagrangian coordinate into a Eulerian coordinate. An alternative method developed by MartínezCasas et al. [3] was deriving the particle velocity with respect to the Eulerian coordinate directly using general dynamic principles. The method for modelling the rotating flexible wheelset used in the current work is originally based on the work in [3] by directly deriving the equations of motion of the wheelset using a virtual work principle.

As far as track models are concerned, besides modelling the rail as Euler-Bernoulli [16] or Timoshenko beam elements [17,18], a recent trend considers modelling the rail as a generic elastically deformable structure. The track model developed by Kaiser in $[12,13]$ consists of a modal synthesis performed on the rails considered as elastically deformable prismatic structures and assumes harmonic waveforms in longitudinal direction. Since the equations of the rails are written in a non-moving reference, this method becomes increasingly computationally expensive when longer lengths travelled by the wheelset are considered. Furthermore, depending on the length of track modelled, the effect of elastic waves travelling in the rails can be affected by the cyclic boundary conditions assumed. For this reason, in this paper a different model is developed for the flexible track, considering an ALE approach. Chamorro et al. [19] developed a Moving Mode Method to describe deformable tracks with arbitrary geometry, an ALE approach is used to define mode shapes of the track. Ripamonti et al. [20] proposed an 'Eulerian' finite element method of the track model, a reasonable number of degrees of freedom can be used due to the flowing finite element mesh along the track. Recently, Martínez-Casas et al. [21] defined a 3D moving element rail model by extending Koh et al.'s Moving Element Method [22] from 2D on continuum to 3D, which is also an extension of the ALE approach. With respect to the approaches 
presented in references [19-22] to model track flexibility in the high-frequency range, this paper additionally addresses the new issue of considering the effect of discrete rail support in the time domain.

Considering wheel/rail contact models in use for the simulation of wheelset-track interaction, the present challenge is to consider detailed capable of accurately predict wear and damage in wheels and rails. Contact models usually can be categorized into Hertzian models [23], non-Hertzian models [24-28] and models based on the finite element method $[29,30]$. Wheel-rail contact models based on the finite element method are however seldom used for train-track interaction studies, due to the very large computational effort implied. The Hertzian model, based on the elliptical contact patch assumption, has been widely used in vehicle-track interaction models in the last decades. However, for real wheel and rail profiles, the contact patch is always non-elliptical and the pressure distribution differs substantially from the one predicted by Hertzian solutions. Approximate non-Hertzian models have therefore been developed to provide a more realistic description of wheel/rail contact conditions, yet requiring a reduced computational effort. Approximate non-Hertzian models include the Kik-Piotrowski model based on virtual penetration concept [24-26] and the STRIPES model proposed by Ayasse and Chollet [28]. Recently, Liu et al. [31] proposed an extended version of the Kik-Piotrowski method which can take into account the effect of yaw and contact patch curvature variation and is proved to be more accurate and efficient comparing with others. However, the application of this kind of contact model in a vehicle-track interaction system is still needed to get a better understanding of dynamic problems arising at the interface of wheel and rail.

The aim of this paper is therefore to present a new train-track interaction model valid in a frequency range up to $7 \mathrm{kHz}$ and defined in the time domain, hence suitable for the investigation of typical problems related with high-frequency train-track interaction such as short pitch rail corrugation and rolling noise. To this aim, an accurate and efficient timedomain model is established by combining a rotating flexible wheelset model, a complete 3D discrete-supported track model and a non-linear, non-Hertzian contact model. The maximum frequency of validity of the model is set to $7 \mathrm{kHz}$ to ensure the model deals satisfactorily with high-frequency wheel-rail interaction problems such as rail corrugation and rolling noise yet keeping to a minimum its computational complexity. The main innovations of the proposed model lie in the modelling of the 3D discretely-supported track and in the use of a non-Hertzian description of wheel/rail contact. The wheelset and the rails are modelled using 3D finite element to ensure a realistic and detailed presentation of system dynamics in high frequency range. The flexible parts are modelled using an ALE approach which describes the model in a moving reference so that on one hand the length of the rail model can be reduced and on the other hand the wheelset and rail matrices are time-independent. Wheel-rail contact is modelled using the Extended Kik-Piotrowski 
method [31] to define the shape and size of the contact patch and the distribution of normal pressures and the FASTSIM algorithm to compute the distribution of tangential stresses.

The results presented in the paper focus on the analysis of the effect of wheelset and track model on the accurate prediction of high frequency effects in train-track interaction. To this aim, results obtained using wheelset and track models having increasing complexity are compared and conclusions on the need to incorporate in the analysis detailed modelling features such as solid FE modelling of the rail and wheelset or modelling of the discrete rail support are drawn depending on the frequency range addressed by the analysis and on the outputs of interest (contact forces, rail vibration).

\section{Mathematical model of the wheelset-track interaction}

While multibody vehicle models based on rigid body assumption are suitable for ride comfort and stability analyses in a frequency range up to $20-30 \mathrm{~Hz}$, high frequency dynamics of train-track interaction is highly influenced by wheelset and track flexibility. This paper aims at the high-frequency range and therefore the vehicle model used here is simplified into one single wheelset whereas the sprung masses of the vehicle (bogie frames, car body) are not considered thanks to the filtering effect provided by vehicle suspensions at sufficiently high frequencies $[3,8,9,32]$. The static forces generated by the weight of the sprung masses is however included in the analysis, as these affect wheel-rail contact. The wheelset and the rail are modelled as 3D finite element bodies with the rail support discretely distributed under the rail foot.

\subsection{Model of the rotating flexible wheelset}

The wheelset model presented here is described as a rotating flexible body, the gyroscopic and inertial effects associated with wheelset rotation are introduced in this model using an ALE finite element approach [3] based on 3D quadratic solid elements. Thanks to the use of the ALE approach, there is no need to consider the changing position of the material contact point at each time step in circumferential direction (whereas the motion of the contact point in transversal direction is accounted for as explained in Section 2.3) and consequently the method is much more efficient during the numerical integration. Moreover, the wheelset's structural matrices are timeindependent so that a modal synthesis of the FE model can be easily performed.

In this work, the wheelset is assumed to move with constant forward speed along the track. Considering an inertial reference XYZ moving with origin in the centre of mass of the undeformed body, $\mathrm{X}$ axis pointing in the direction of forward movement $\mathrm{Y}$ axis lateral and $\mathrm{Z}$ axis vertical pointing upwards, the position vector $\mathbf{s}$ of a material point in the wheelset can be expressed as: 


$$
\mathbf{S}=\mathbf{u}+\mathbf{w}(\mathbf{u}, t)
$$

with $\mathbf{u}$ the position of the material point in the wheelset's undeformed configuration and $\mathbf{w}(\mathbf{u}, t)$ the displacement associated with wheelset flexibility, which is assumed to be small. Fig. 1 shows the wheelset coordinate system and the displacement vectors of a wheelset point.

The wheelset is modelled according to the Finite Element Method. To reduce the computational cost of the model, a modal synthesis is introduced. In this way, the nonrigid part of the displacement in e-th element in volume $v^{e}$ is:

$$
\mathbf{w}^{e}(\mathbf{u}, t)=\mathbf{N}^{e}(\mathbf{u}) \mathbf{x}^{e}(t)=\mathbf{N}^{e}(\mathbf{u}) \Phi^{e}(\mathbf{u}) \mathbf{q}(t) \quad \mathbf{u} \in v^{e},
$$

where $\mathbf{N}^{\mathrm{e}}(\mathbf{u})$ is the shape function matrix of the e-th element, $\mathbf{x}^{\mathrm{e}}(t)$ is the nodal displacement of the element, $\boldsymbol{\Phi}^{e}(\mathbf{u})$ is a matrix containing the mode shapes for the nodes of the e-th element which are computed from the finite element model of the wheelset, and $\mathbf{q}(t)$ is the modal coordinate vector.

The derivation of the wheelset's equations of motion is presented in [3] and results in the following equation of motion in matrix form:

$$
\ddot{\mathbf{q}}+2 \Omega \boldsymbol{\Phi}_{F E, w}^{T} \mathbf{V} \boldsymbol{\Phi}_{F E, w} \dot{\mathbf{q}}+\boldsymbol{\Phi}_{F E, w}^{T}\left(\mathbf{K}+\Omega^{2}(\mathbf{A}-\mathbf{C})\right) \boldsymbol{\Phi}_{F E, w} \mathbf{q}=\Omega^{2} \boldsymbol{\Phi}_{F E, w}^{T} \mathbf{L}+\mathbf{Q}_{c w}
$$

where $\boldsymbol{\Phi}_{F E, w}$ is the matrix obtained from the assembling of the $\Phi^{e}$ matrices over the entire FE model of the wheelset, $\Omega$ is the angular speed of the wheelset related to its rolling over the rails, $\mathbf{V}, \mathbf{A}, \mathbf{C}$ and $\mathbf{L}$ are the matrices obtained from the assembling of matrices $\mathbf{V}^{\mathrm{e}}, \mathbf{A}^{\mathrm{e}}, \mathbf{C}^{\mathrm{e}}, \mathbf{L}^{\mathrm{e}}$ related to the gyroscopic and inertial effects associated with wheelset rotation and defined as follows:

$$
\begin{aligned}
& \mathbf{V}^{e}=\int_{v^{e}} \rho \mathbf{N}^{e T} \sum_{i=1}^{3} \tilde{u}_{i} \frac{\partial \mathbf{N}^{e}}{\partial u_{i}} d v ; \\
& \mathbf{C}^{e}=\int_{v^{e}} \rho \mathbf{N}^{e T} \sum_{i=1}^{3} \mathbf{E} u_{i} \frac{\partial \mathbf{N}^{e}}{\partial u_{i}} d v ; \\
& \mathbf{A}^{e}=\int_{v^{e}} \rho \mathbf{N}^{e T} \sum_{i=1}^{3} \sum_{j=1}^{3} \tilde{u}_{i} \tilde{u}_{j} \frac{\partial^{2} \mathbf{N}^{e}}{\partial u_{i} \partial u_{j}} d v ; \\
& \mathbf{L}^{e}=\int_{v^{e}} \rho \mathbf{N}^{e T} \sum_{i=1}^{3} \mathbf{E} u_{i} d v ;
\end{aligned}
$$

Finally, $\mathbf{Q}_{c w}$ is the vector of generalized forces due to wheel-rail contact. Note that the normalization of the modal shapes is such that a unit mass matrix is obtained.

In this work, a S1002 wheelset profile is modelled using ABAQUS (Fig. 2). 3D solid elements with 20 nodes are used in the mesh, which can describe the bending motion better than linear solid element. More details can be found in the authors' previous work [32]. 200 modes of the wheelset are chosen to cover a system frequency up to $7 \mathrm{kHz}$. 


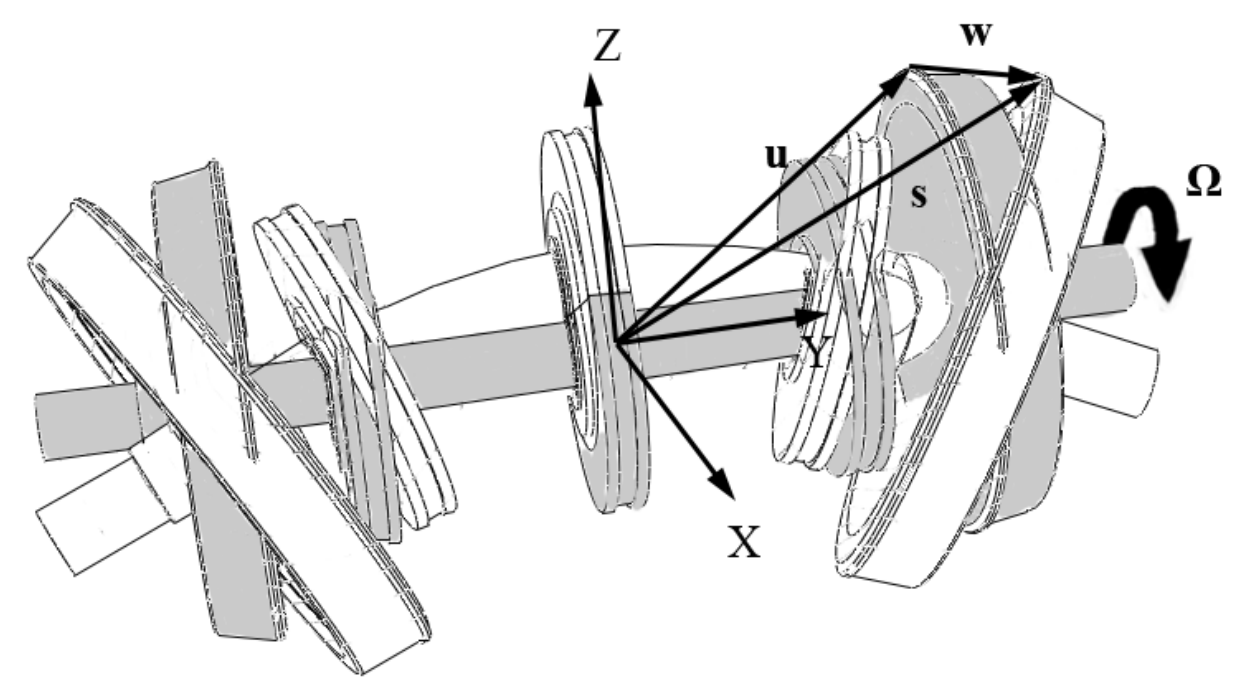

Figure 1. Wheelset reference system, undeformed position vector $\mathbf{u}$ and displacement due to flexibility $\mathbf{w}$.

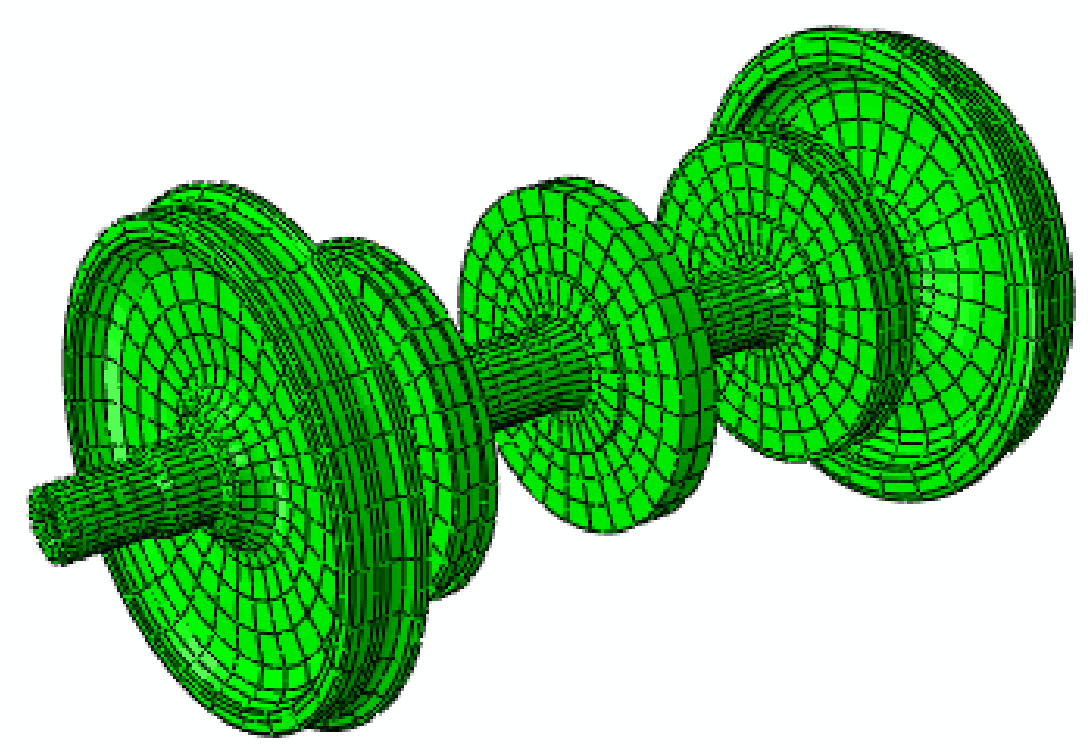

Figure 2. Finite element model of the wheelset.

\subsection{Model of the track}

The track model presented here is described as a 3D ALE finite element model with discrete support to obtain a detailed representation of rail vibration in the high frequency range. Using an ALE approach, the rail mesh travels with the contact point so it is possible not only to use a shorter model of the rail, but also the calculation of wheel/rail contact forces is simplified and results in a more efficient process from a computational point of view. A discrete visco-elastic support is added to the rail in order to introduce the nonlinear dynamic effect of the rail support. 


\subsubsection{Model of the rail}

The finite element model of the rail is meshed using ABAQUS (Fig. 3). Eight node hexahedral solid elements are used in the discretization. The finite element mesh is considered to move across the rail volume, with the same constant speed $V$ as the wheelset centre of mass. The position vector $\mathbf{r}$ of a material point in the rail in an ALE reference system can be expressed as:

$$
\mathbf{r}=\mathbf{u}+\mathbf{w}(\mathbf{u}, t)
$$

where $\mathbf{u}$ is the undeformed position of the point which is independent on time, and $\mathbf{w}(\mathbf{u}, t)$ is the displacement associated with rail flexibility of position $u$ at time $t$. According to a traditional finite element formulation the vector $\mathbf{w}(\mathbf{u}, t)$ of material displacement in the i-th finite element is:

$$
\mathbf{w}(\mathbf{u}, t)=\mathbf{N}_{i}(\mathbf{u}) \mathbf{x}(t)
$$

where $\mathbf{N}_{\mathbf{i}}(\mathbf{u})$ is the shape function matrix of the element and $\mathbf{x}(t)$ is the vector of nodal displacements for the entire rail model. Because of the formulation in the moving reference, time-dependency is introduced to the shape functions matrix of the rail:

$$
\mathbf{N}_{i}(\mathbf{u})=\mathbf{N}_{i}\left(u_{1}-V t, u_{2}, u_{3}\right)
$$

The velocity and acceleration of the material point take therefore the following expressions:

$$
\begin{aligned}
\dot{\mathbf{r}} & =\frac{D \mathbf{r}}{D t}=\frac{D \mathbf{u}}{D t}+\frac{D \mathbf{w}}{D t}=\left(\begin{array}{lll}
-V & 0 & 0
\end{array}\right)^{T}+\mathbf{N}_{i}(\mathbf{u}) \dot{\mathbf{x}}-V \frac{\partial \mathbf{N}_{i}(\mathbf{u})}{\partial u_{1}} \mathbf{x} \\
\ddot{\mathbf{r}} & =\frac{D^{2} \mathbf{r}}{D t^{2}}=\mathbf{N}_{i}(\mathbf{u}) \ddot{\mathbf{x}}-2 V \frac{\partial \mathbf{N}_{i}(\mathbf{u})}{\partial u_{1}} \dot{\mathbf{x}}+V^{2} \frac{\partial^{2} \mathbf{N}_{i}(\mathbf{u})}{\partial u_{1}{ }^{2}} \mathbf{x}
\end{aligned}
$$

The equation of motion of the track model is deduced using Lagrange's equation. According to [33], Lagrange's equations written with respect to a non-material volume subjected to material flow read:

$$
\frac{d}{d t}\left(\frac{\partial E_{k}}{\partial \dot{\mathbf{x}}}\right)^{T}-\left(\frac{\partial E_{k}}{\partial \mathbf{x}}\right)^{T}+\int_{S}\left(\frac{\delta E_{k}^{\prime \prime}}{\delta \dot{\mathbf{x}}}\right)^{T} \mu d S-\int_{S} E_{k}^{\prime \prime}\left(\frac{\delta \mu}{\delta \dot{\mathbf{x}}}\right)^{T} d S=\mathbf{F}
$$

Where the last two terms in the left-hand side of the equation are added with respect to the usual Lagrange's equations for a system with no mass flow. In (10) $S$ is the surface across which mass flow takes place (control surface), which consists in this case of the leftmost and rightmost rail sections delimiting the control volume, $E_{k}^{\prime \prime}$ is the kinetic 
energy per unit mass:

$$
E_{k}^{\prime \prime}=\frac{1}{2} \dot{\mathbf{r}}^{T} \dot{\mathbf{r}}
$$

and $\mu$ is the flow of mass across the surface of the control volume:

$$
\mu=\left(\operatorname{det}\left(\frac{\partial \mathbf{r}}{\partial \mathbf{u}}\right)\right)^{-1} \rho\left(\dot{\mathbf{r}}-\mathbf{v}_{s}\right)^{T} \mathbf{n}
$$

In Eq. (11) and (12) $\dot{\mathbf{r}}$ is the material velocity of the particles inside the control volume and $\mathbf{v}_{s}$ is the speed of the control surface, which is in our case uniform at all surface points:

$$
\mathbf{v}_{s}=\left\{\begin{array}{lll}
V & 0 & 0
\end{array}\right\}^{T}
$$

We notice that the amplitude of the particle's material velocity rapidly decays with the distance from the point of contact with the wheel, as the region of the rail interested by bending and torsional deformation being in the order of few multiples of the sleeper bay $(0.6 \mathrm{~m})$ whilst the model considered here extend over a length of $48 \mathrm{~m}$. Hence, at the two extremities of the control volume where mass flow takes place the kinetic energy per unit mass $E_{k}^{\prime \prime}$ is negligible so that the third term in the left-hand side of Eq. (10) is negligible. Furthermore, in the case considered here the component along the longitudinal direction $x$ of the material velocity of particles $\dot{\mathbf{r}}$ is zero because axial movements of the rails are not included in the analysis. It follows that on the entire control surface:

$$
\dot{\mathbf{r}}^{T} \mathbf{n}=0
$$

while the product $\mathbf{v}_{s}{ }^{T} \mathbf{n}$ is either $\pm V$ depending on which end of the control volume is considered. Therefore, denoting by $S_{l}$ the surface of the rail section we have:

$$
\begin{aligned}
& \int_{S}\left(\frac{\delta E_{k}^{\prime \prime}}{\delta \dot{\mathbf{x}}}\right)^{T} \mu d S=\int_{S}\left(\frac{\delta E_{k}^{\prime \prime}}{\delta \dot{\mathbf{x}}}\right)^{T}\left(\operatorname{det}\left(\frac{\partial \mathbf{r}}{\partial \mathbf{u}}\right)\right)^{-1} \rho\left(\dot{\mathbf{r}}-\mathbf{v}_{s}\right)^{T} \mathbf{n} d S= \\
& =\int_{S_{1}}\left(\frac{\delta E_{k}^{\prime \prime}}{\delta \dot{\mathbf{x}}}\right)^{T}\left(\operatorname{det}\left(\frac{\partial \mathbf{r}}{\partial \mathbf{u}}\right)\right)^{-1} \rho(-V) d S+\int_{S_{1}}\left(\frac{\delta E_{k}^{\prime \prime}}{\delta \dot{\mathbf{x}}}\right)^{T}\left(\operatorname{det}\left(\frac{\partial \mathbf{r}}{\partial \mathbf{u}}\right)\right)^{-1} \rho V d S=0
\end{aligned}
$$


Considering the above results and re-writing the generalized force vector $\mathbf{F}$ as the sum of four terms representing respectively the effect of elastic forces (described as the partial derivative with respect to vector $\mathbf{x}$ of the potential energy $E_{p}$ ), the viscous damping forces (described as the partial derivative with respect to vector $\dot{\mathbf{x}}$ of the dissipation function $D$ ), the generalized forces due to wheel-rail contact $\mathbf{F}_{c t}$ and the generalized forces $\mathbf{F}_{s}$ due to the effect of discrete rail support (see section 2.2.2):

$$
\mathbf{F}=-\left(\frac{\partial E_{p}}{\partial \mathbf{x}}\right)^{T}-\left(\frac{\partial D}{\partial \dot{\mathbf{x}}}\right)^{T}+\mathbf{F}_{c t}(t)+\mathbf{F}_{s}(t)
$$

Neglecting the third term in the left-hand side of Eq. (10) for the reasons stated above, and considering Eq.s (15) and (16), Lagrange's equations for the rail modelled according to the ALE approach are finally obtained:

$$
\frac{d}{d t}\left(\frac{\partial E_{k}}{\partial \dot{\mathbf{x}}}\right)^{T}-\left(\frac{\partial E_{k}}{\partial \mathbf{x}}\right)^{T}+\left(\frac{\partial E_{p}}{\partial \mathbf{x}}\right)^{T}+\left(\frac{\partial D}{\partial \dot{\mathbf{x}}}\right)^{T}=\mathbf{F}_{c t}(t)+\mathbf{F}_{s}(t)
$$

For the 3D solid element rail model, the expression of the kinetic energy is:

$$
E_{k}=\frac{1}{2} \sum_{i=1}^{N_{e}} \int_{v_{i}} \rho \frac{\mathrm{D} \mathbf{r}^{\mathrm{T}}}{\mathrm{D} t} \frac{\mathrm{Dr}}{\mathrm{D} t} d v=\frac{1}{2} \dot{\mathbf{x}}^{\mathrm{T}} \sum_{i=1}^{N_{e}} \int_{v_{i}} \mathbf{N}_{i}^{T} \rho \mathbf{N}_{i} d v \dot{\mathbf{x}}-V \mathbf{x}^{\mathrm{T}} \sum_{i=1}^{N_{c}} \int_{v_{i}} \frac{\partial \mathbf{N}_{i}^{T}}{\partial u_{1}} \rho \mathbf{N}_{i} d v \dot{\mathbf{x}}+\frac{1}{2} V^{2} \mathbf{x}^{\mathrm{T}} \sum_{i=1}^{N_{e}} \int_{v_{i}} \frac{\partial \mathbf{N}_{i}^{T}}{\partial u_{1}} \rho \frac{\partial \mathbf{N}_{i}}{\partial u_{1}} d v \mathbf{x}
$$

The potential energy of the rail model is:

$$
E_{p}=\frac{1}{2} \mathbf{x}^{\mathrm{T}} \mathbf{K}_{\mathrm{r}} \mathbf{x}
$$

with $\mathbf{K}_{\mathbf{r}}$ the stiffness matrix of the rail model obtained from a standard finite element method.

Since the flow of the mesh through the material coordinates does not change the mode shapes in spatial coordinates, the mode shapes of the rail model do not depend on time, which makes it possible to use modal synthesis during the simulation:

$$
\mathbf{x}=\mathbf{\Phi}_{F E, r} \mathbf{q}
$$

where $\mathbf{q}$ and $\boldsymbol{\Phi}_{F E, r}$ are the modal coordinates and the modal matrix of the finite element rail model, respectively.

Introducing in Eq. (18) the following notation: 


$$
\begin{aligned}
& \mathbf{M}_{\mathrm{r}}=\sum_{i=1}^{N_{e}} \int_{v_{i}} \mathbf{N}_{i}^{T} \rho \mathbf{N}_{i} d v ; \\
& \mathbf{D}_{\mathrm{r}}=\sum_{i=1}^{N_{e}} \int \mathbf{N}_{v_{i}}^{T} \rho \frac{\partial \mathbf{N}_{i}^{T}}{\partial u_{1}} d v ; \\
& \mathbf{A}_{\mathrm{r}}=\sum_{i=1}^{N_{e}} \int \frac{\partial \mathbf{N}_{i}^{T}}{\partial u_{1}} \rho \frac{\partial \mathbf{N}_{i}}{\partial u_{1}} d v ;
\end{aligned}
$$

and considering Eq. (20), the expression of the kinetic energy becomes:

$$
E_{k}=\frac{1}{2} \dot{\mathbf{q}}^{\mathrm{T}} \boldsymbol{\Phi}_{F E, r}^{T} \mathbf{M}_{r} \boldsymbol{\Phi}_{F E, r} \dot{\mathbf{q}}-V \mathbf{q}^{\mathrm{T}} \boldsymbol{\Phi}_{F E, r}^{T} \mathbf{D}_{r}^{T} \boldsymbol{\Phi}_{F E, r} \dot{\mathbf{q}}+\frac{1}{2} V^{2} \mathbf{q}^{\mathrm{T}} \boldsymbol{\Phi}_{F E, r}^{T} \mathbf{A}_{r} \boldsymbol{\Phi}_{F E, r} \mathbf{q}
$$

whilst the expression of the potential energy becomes:

$$
E_{p}=\frac{1}{2} \mathbf{q}^{\mathbf{T}} \boldsymbol{\Phi}_{F E, r}^{T} \mathbf{K}_{\mathrm{r}} \boldsymbol{\Phi}_{F E, r}^{T} \mathbf{q}=\frac{1}{2} \mathbf{q}^{\mathbf{T}} \tilde{\mathbf{K}}_{\mathrm{r}} \mathbf{q}
$$

A structural damping term is introduced for the rail in the form:

$$
D==\frac{1}{2} \dot{\mathbf{q}}^{\mathrm{T}} \tilde{\mathbf{C}}_{\mathrm{r}} \dot{\mathbf{q}}
$$

with the modal damping matrix $\tilde{\mathbf{C}}_{\mathrm{r}}$ defined as:

$$
\tilde{\mathbf{C}}_{\mathrm{r}}=2 \zeta \sqrt{\mathbf{\Phi}_{F E, r}^{T} \mathbf{K}_{\mathrm{r}} \boldsymbol{\Phi}_{F E, r}}
$$

the loss factor $\zeta$ being set to $\zeta=0.012$.

Considering Eq.s (22) to (24) and assuming a normalization of the mode shapes that provides a unit mass matrix, the left hand side of the Lagrange's equation for the rail in the new set of modal coordinates $\mathbf{q}$ reads:

$$
\frac{d}{d t}\left(\frac{\partial E_{k}}{\partial \dot{\mathbf{q}}}\right)^{T}-\left(\frac{\partial E_{k}}{\partial \mathbf{q}}\right)^{T}+\left(\frac{\partial E_{p}}{\partial \mathbf{q}}\right)^{T}+\left(\frac{\partial D}{\partial \dot{\mathbf{q}}}\right)^{T}=\ddot{\mathbf{q}}+\left(\tilde{\mathbf{C}}_{\mathrm{r}}-2 V \boldsymbol{\Phi}_{F E, r}^{T} \mathbf{D}_{\mathrm{r}} \boldsymbol{\Phi}_{F E, r}\right) \dot{\mathbf{q}}+\boldsymbol{\Phi}_{F E, r}^{T}\left(\tilde{\mathbf{K}}_{\mathrm{r}}-V^{2} \mathbf{A}_{\mathrm{r}}\right) \boldsymbol{\Phi}_{F E, r} \mathbf{q}
$$

The length considered for the track model is $48 \mathrm{~m}$. Both rails are modelled, each one coming in contact with one of the two wheels. For each rail, 1000 modes are considered to cover a system frequency up to $7 \mathrm{kHz}$. Fig. 4 compared the vertical receptance of the rail obtained from the modal superimposition to the result directly obtained from the FE model using ABAQUS. The modal rail modes are nearly the same with the direct rail receptance obtained from the FE model, indicating that the modal synthesis can successfully describe the rail flexibility up to $7 \mathrm{kHz}$. By using such modes, deformations of the cross-section can be described in addition to the usual deformations 
such as bending or torsion. Examples of the rail modes are shown in Fig. 5.

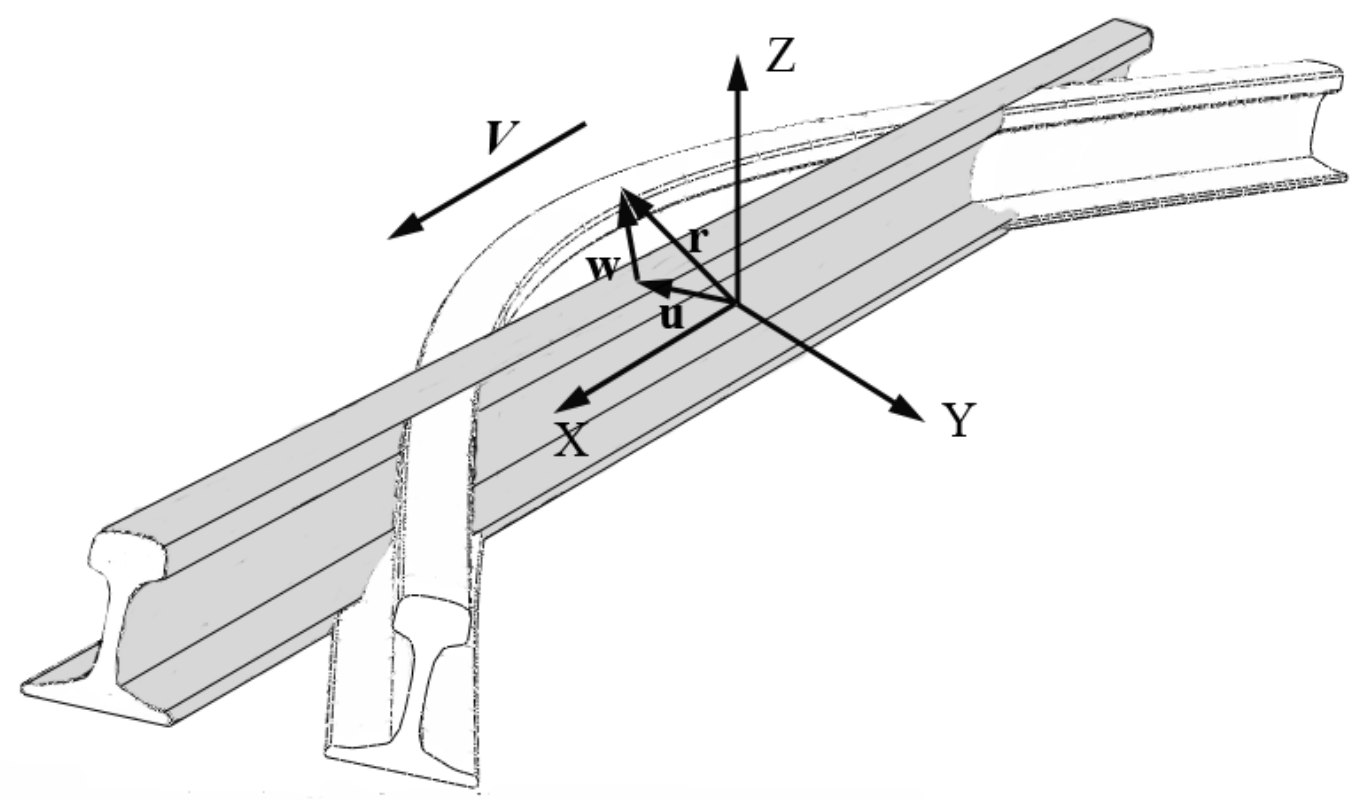

Figure 3. Reference system of the rail, undeformed position vector $\mathbf{u}$ and displacement due to flexibility $\mathbf{w}$.

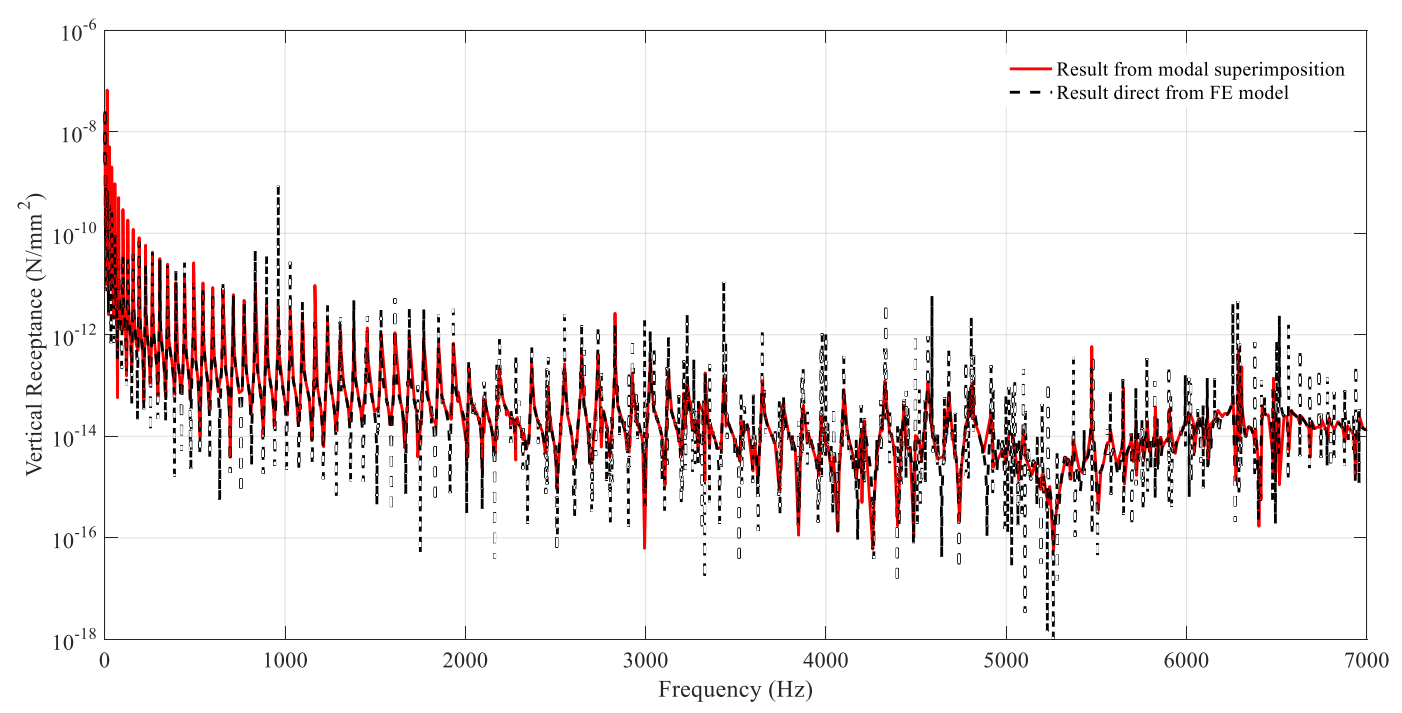

Figure 4. Direct rail receptance at the contact point from modal superimposition and direct FE model. 


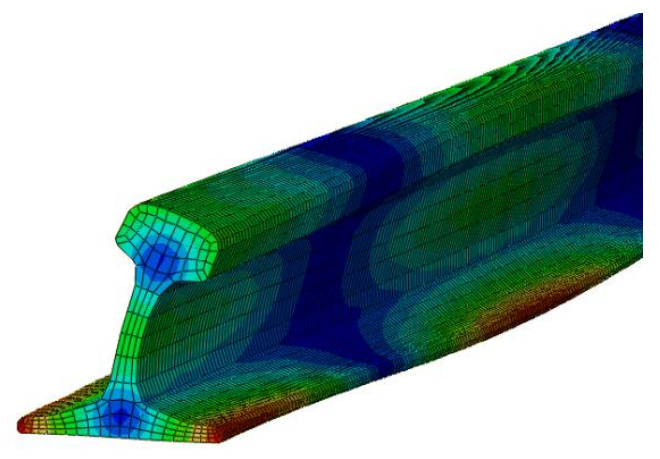

(a)

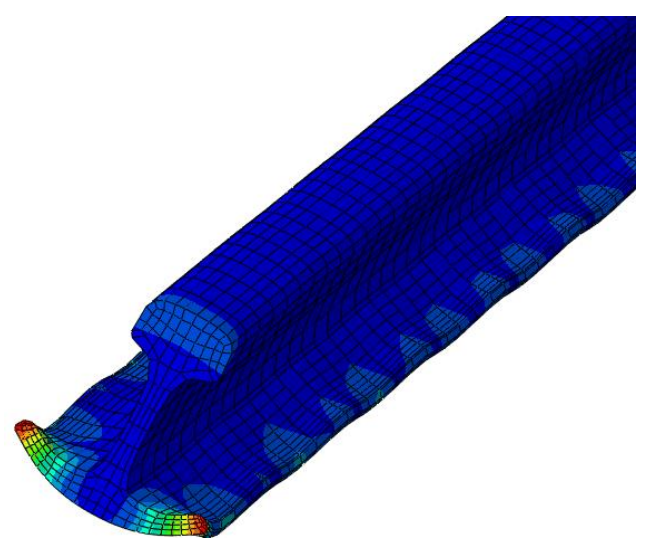

(c)

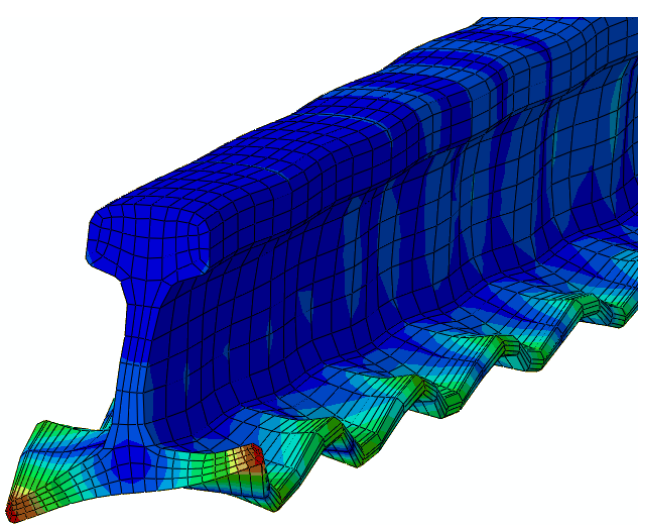

(b)

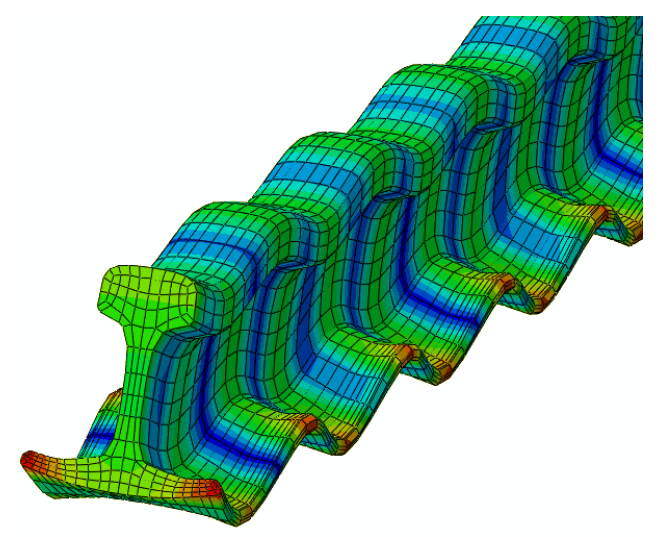

(d)

Figure 5. Rail modes: (a) Rail web bending at $1530 \mathrm{~Hz}$; (b) Rail foot rocking at $2576 \mathrm{~Hz}$; (c) Rail foot flapping at $3078 \mathrm{~Hz}$; (d) Rail foot flapping at $3098 \mathrm{~Hz}$.

\subsubsection{Model of the rail support}

Considering the discrete nature of rail support is pivotal to reproducing some train-track interaction phenomena in the high frequency range, as the discrete support affects the natural frequencies of the rail, e.g. the so-called pinned-pined resonance $[34,35]$ and the periodic variation of rail impedance is one of the mechanisms triggering the formation of rail corrugation [36]. In this work, the rail supports are represented as distributed linear visco-elastic elements set under the rail foot. The elastic energy and dissipative function related with all these elements read:

$$
\begin{gathered}
E_{p, \text { sup }}=\sum_{i=1}^{N_{\text {sup }}} \frac{1}{2} \int_{s_{i}} \mathbf{w}^{T} \boldsymbol{\beta} \mathbf{w} d s_{i}=\frac{1}{2} \mathbf{x}^{T}\left(\sum_{i=1}^{N_{\text {sup }}} \int_{s_{i}} \mathbf{N}_{i}^{T} \boldsymbol{\beta} \mathbf{N}_{i} d s_{i}\right) \mathbf{x} \\
D_{\text {sup }}=\frac{1}{2} \sum_{i=1}^{N_{\text {sup }}} \int\left(\frac{\partial \mathbf{w}}{\partial t}\right)^{T} \boldsymbol{\eta}\left(\frac{\partial \mathbf{w}}{\partial t}\right) d s \\
=\frac{1}{2} \dot{\mathbf{x}}^{T}\left(\sum_{i=1}^{N_{\text {sup }}} \int \mathbf{N}_{s_{i}}^{T} \boldsymbol{\eta} \mathbf{N}_{i} d s_{i}\right) \dot{\mathbf{x}}-V \mathbf{x}^{T}\left(\sum_{i=1}^{N_{\text {spp }}} \int_{s_{i}} \frac{\partial \mathbf{N}_{i}^{T}}{\partial u_{1}} \boldsymbol{\eta} \mathbf{N}_{i} d s_{i}\right) \dot{\mathbf{x}}+\frac{1}{2} V^{2} \mathbf{x}^{T}\left(\sum_{i=1}^{N_{\text {sup }}} \int_{s_{i}} \frac{\partial \mathbf{N}_{i}^{T}}{\partial u_{1}} \boldsymbol{\eta} \frac{\partial \mathbf{N}_{i}}{\partial u_{1}} d s_{i}\right) \mathbf{x}
\end{gathered}
$$


where $N_{\text {sup }}$ is the number of finite elements in the rail foot coming in contact with a rail pad, $s_{i}$ is the portion of the i-th element's lower face contacting the pad (see below), $\boldsymbol{\beta}$ and $\boldsymbol{\eta}$ are square matrices of order 3 defined as:

$$
\boldsymbol{\beta}=\operatorname{diag}\left[\begin{array}{lll}
\beta_{n} & \beta_{s} & \beta_{n}
\end{array}\right] \quad ; \quad \boldsymbol{\eta}=\operatorname{diag}\left[\begin{array}{lll}
\eta_{s} & \eta_{s} & \eta_{n}
\end{array}\right]
$$

with $\beta_{n}$ and $\beta_{s}$ the bulk modulus (stiffness per unit area) of the pad in normal and shear direction respectively, and $\eta_{n}, \eta_{s}$ the damping coefficient per unit area of the pad in normal and shear direction.

To simplify the notation in Eq. (32), we introduce matrices $\mathbf{K}_{\text {sup }}, \mathbf{C}_{\text {sup }}$ defined as:

$$
\begin{aligned}
& \mathbf{K}_{\text {sup }}=\sum_{i=1}^{N_{\text {sup }}} \int_{s_{i}} \mathbf{N}_{i}^{T} \boldsymbol{\beta} \mathbf{N}_{i} d s_{i}-V \sum_{i=1}^{N_{\text {sup }}} \int_{s_{i}} \mathbf{N}_{i}^{T} \boldsymbol{\gamma} \frac{\partial \mathbf{N}_{i}}{\partial u_{1}} d s_{i} ; \\
& \mathbf{C}_{\text {sup }}=\sum_{i=1}^{N_{\text {sup }}}\left(\int_{s_{i}} \mathbf{N}_{i}^{T} \boldsymbol{\gamma} \mathbf{N}_{i} d s_{i}\right) ;
\end{aligned}
$$

Introducing the modal approach in Eq.s (31) and (32) and applying Lagrange's derivatives, the following terms are obtained for the generalized forces applied on the rail by the discrete supports:

$$
\left(\frac{\partial D_{\text {sup }}}{\partial \dot{\mathbf{q}}}\right)^{T}+\left(\frac{\partial E_{\text {sup }}}{\partial \mathbf{q}}\right)^{T}=\boldsymbol{\Phi}_{F E, r}^{T} \mathbf{K}_{\text {sup }} \boldsymbol{\Phi}_{F E, r} \mathbf{q}+\boldsymbol{\Phi}_{F E, r}^{T} \mathbf{C}_{\text {sup }} \boldsymbol{\Phi}_{F E, r} \dot{\mathbf{q}}
$$

Due to the discrete nature of the rail support and to the ALE model of the rail, matrices $\mathbf{K}_{\text {sup }}$ and $\mathbf{C}_{\text {sup }}$ are time dependent and need to be re-computed at each time step. To this aim, at a given time step a search is performed of the finite elements in the rail foot being in contact with a rail pad and for each one of these the rectangular portion of the bottom face of the element in contact with the pad is found. This consists of the entire width of the element in $\mathrm{Y}$ direction (see Fig. 3) and of a region of X values ranging from $a$ to $b$, being these values the X coordinates of the leading and trailing edge of the rectangular portion as shown in Fig. 6. To speed-up the process of computing matrices $\mathbf{K}_{\text {sup }}$ and $\mathbf{C}_{\text {sup }}$ the surface integrals in Eq. (30) are computed in closed form as a function of parameters $a$ and $b$, thanks to the simple analytical expression taken by the shape functions on the bottom face of the element. 


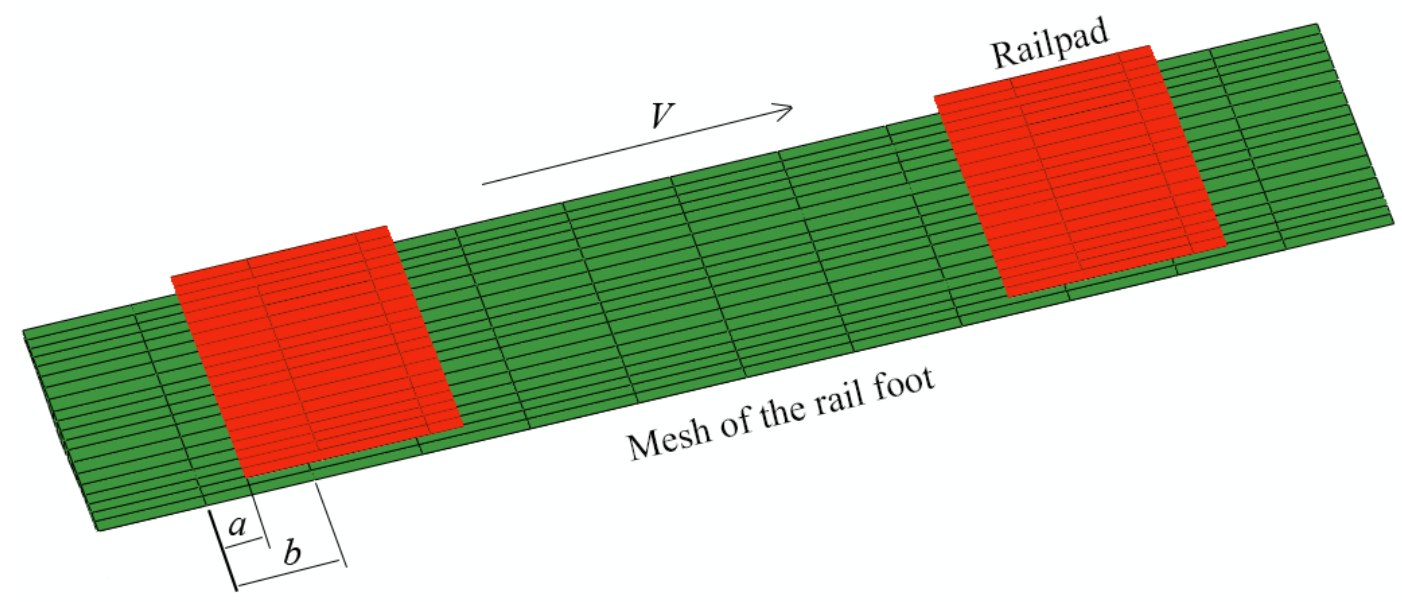

Figure 6. Discrete support under the mesh of rail foot ( $a$ and $b$ indicate the position of the support with respect to one element).

In order to avoid time-varying structural matrices for the rail model, the stiffness and damping terms arising from the discrete rail supports are moved to the right hand side of the equations, in the form a system of visco-elastic forces $\mathbf{F}_{\mathrm{s}}$ :

$$
\mathbf{F}_{s}=-\boldsymbol{\Phi}_{F E, r}^{T} \mathbf{C}_{\text {sup }}(t) \boldsymbol{\Phi}_{F E, r} \dot{\mathbf{q}}-\boldsymbol{\Phi}_{F E, r}^{T} \mathbf{K}_{\text {sup }}(t) \boldsymbol{\Phi}_{F E, r} \mathbf{q}
$$

Considering Eq.s (26) and (32), the equations of motion of the track model read as follows:

$$
\ddot{\mathbf{q}}+\left(\tilde{\mathbf{C}}_{r}-2 V \boldsymbol{\Phi}_{F E, r}^{T} \mathbf{D}_{r} \boldsymbol{\Phi}_{F E, r}\right) \dot{\mathbf{q}}+\boldsymbol{\Phi}_{F E, r}^{T}\left(\mathbf{K}_{r}-V^{2} \mathbf{A}_{r}\right) \boldsymbol{\Phi}_{F E, r} \mathbf{q}=\mathbf{F}_{c t}+\mathbf{F}_{s}
$$

With $\mathbf{F}_{\mathrm{ct}}$ the vector of generalized forces arising from wheel/rail contact (cf. Section 2.3).

\subsection{Wheel-rail contact model}

Prior to the simulation, the analysis of wheel-rail contact geometry for different positions of the lateral shift of the wheelset relative to the rails is performed, considering theoretical S1002 wheel profiles and UIC60 rail profiles with 1:40 inclination. From this analysis, the contact parameters required to compute wheel-rail contact forces are stored in a contact table [37]. Contact parameters include the contact angle, the variation of the wheel rolling radius with respect to the nominal radius and the curvatures of the wheel and rail profiles in the contact point region.

Considering the use of the ALE approach, the position of the geometric contact point on the rolling surfaces is not changing in circumferential direction on the wheel and the same happens for the longitudinal position of the contact point on the rail. However, the transversal movement of the contact point on the wheel and rail surfaces is 
considered in the calculation of wheel/rail contact forces. To this aim, the relative wheel/rail displacement in lateral direction is determined for each time instant, the contact table is then used to derive the transversal position of the contact point as a function of the relative wheel/rail displacement in lateral direction; note that in doing so, the effect of wheelset and rail deformability on the transversal movement of the contact point is neglected. This is however an acceptable approximation, as the motion of the contact point across the wheel and rail transversal profile is mostly due to the rigid relative motion of the wheel over the rail which is allowed by the gap existing between the wheel flange and the inner side of the rail.

Once the transversal position of the contact point is determined, the displacement and velocity of the contact point on the wheel and rail surfaces is obtained by means of linear interpolation of the nodal displacements. Then, the relative wheel/rail displacement and velocity in the contact point obtained from each time step is projected along the normal and tangential directions using the local inclination of the tangent plane (contact angle) coming from the contact table. The relative displacement in normal direction is used as the normal approach $\delta$ to compute the normal contact force, while the components of the relative wheel/rail velocity in the tangent plane are used to derive the creepage components used to derive the tangential stresses and forces, see below.

\subsubsection{Solutions of the normal and tangential contact problems}

To get a realistic representation of wheel/rail contact in the simulation, the ExtendedKik-Piotrowski (EKP) method [31] is used in the wheelset-track interaction model. This is a generalization of the Kik-Piotrowski algorithm [26] and is capable of representing non-Hertzian contact conditions between the wheel and the rail also considering the effect of a relative yaw rotation of the profiles.

The EKP method is a fast and non-iterative method based on virtual penetration hypothesis. When the undeformed surfaces of wheel and rail at the contact point are shifted towards each other by a distance $\delta$, the EKP method assumes they are virtually penetrated to a depth $\delta_{0}=0.5 \delta$ and the resulting projection region of the interpenetration corrected by a scaling factor $k$ is taken as an approximation of the actual contact zone. The scaling factor has been determined on the basis of numerous tests carried out using program CONTACT and finally a value of 0.5 showed a good agreement with program CONTACT in [31]. The contact patch is then partitioned into strips parallel to the direction of wheel rolling, which is chosen as the $x$-axis of a local reference defined in the contact plane. The normal force and maximum pressure over the contact patch are expressed as: 


$$
\begin{aligned}
& N=\frac{\pi E \delta}{2\left(1-\sigma^{2}\right)} \cdot \frac{\int_{y_{r}}^{y_{l}} x_{-x_{t}}^{x_{l}} \sqrt{x_{l}^{2}(y)-x^{2}} d x d y}{\int_{y_{r}}^{y_{l}} \int_{-x_{t}}^{x_{l}} \frac{\sqrt{x_{l}^{2}(y)-x^{2}}}{\sqrt{x^{2}+y^{2}}} d x d y} ; \\
& p_{0}=\frac{N \sqrt{2 R \delta_{0}}}{\int_{y_{r}}^{y_{l}} \int_{-x_{t}}^{x_{l}} \sqrt{x_{l}^{2}(y)-x^{2}} d x d y}
\end{aligned}
$$

where $x_{l}, x_{t}$ represents the leading and trailing edges of the contact patch in $x$ direction and $y_{l}, y_{r}$ represents the left and right edges of the contact patch in $y$ direction. $R$ is the rolling radius of the wheel. In order to estimate the normal pressure distribution over the contact patch, the strips in the contact zone are divided into $m \times n$ cells. For each cell the pressure is assumed to be constant and is determined according to the following formula:

$$
p(x, y)=\left(1-\frac{1}{k}\right) \frac{p_{0}}{x_{l}(0)} \sqrt{x_{l}^{2}(y)-x^{2}}
$$

The tangential problem is solved using the FASTSIM algorithm [38] by Kalker. The flexibility parameter $L$ used by the FASTSIM algorithm to define the tangential stresses in the adhesion region is obtained from an equivalent ellipse defined by setting the ellipse area equal to the area of the non-elliptic contact patch and the ellipse semi-axes ratio equal to length to width ratio of the patch [26]. The tangential stresses are calculated from the creepages at the contact patch. Slip occurs in the region where the tangential stresses predicted are greater than the traction bound. The formulation for the traction bound used in this paper is obtained by applying Coulomb's friction law locally with a constant friction coefficient. The tangential forces are finally obtained from the numerical integration of the stresses over the contact patch.

\subsubsection{Optimization of contact patch discretisation}

The EKP method together with the FASTSIM solution to wheel-rail contact is proved to be very fast in [31] for a local contact calculation with the contact patch divided into 53 strips for both the $x$ and the $y$ direction. However, the number of strips to be used for discretizing the contact patch needs to be carefully evaluated when tens of thousands time steps are considered in an on-line simulation of the wheelset-track interaction. In order to satisfy both accuracy and time efficiency, the wear number per unit area ( $\mathrm{Wn}=\mathrm{T} \gamma / \mathrm{A})$ [39] of each single contact with different numbers of strips on the contact patch was investigated, see Fig. 6 . In this analysis, the tangential creepages are assumed to be $0.1 \%$. [SB1][s2]In Fig. 7, a smoothing spline interpolation of the points is used to show the general trend of the wear number with the increase of the number of strips. The convergence of the wear number seems to be obtained already using 15 strips. However, for a better evaluation of the contact condition, a conservative choice of using 19 strips in the lateral direction of the contact plane is chosen for the on-line simulation. 


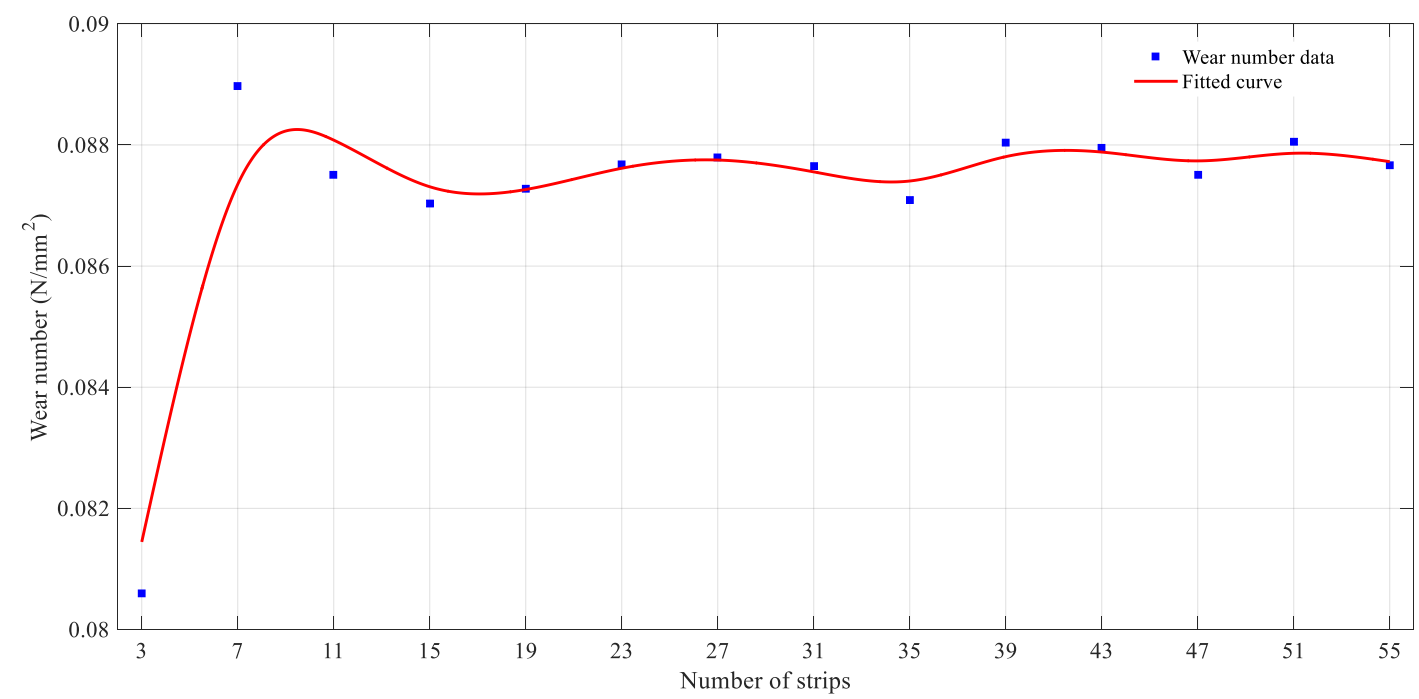

Figure 7. Effect of the number of strips in the lateral direction of the contact plane on wear number $(0.1 \%$ creepage).

A typical contact condition between S1002 wheel profile and 1:40 inclined UIC60 rail profile described by EKP method is shown in Fig. 8, where the contact patch is clearly non-elliptical and the maximum pressure occurs at the left end of the contact patch, which would definitely influence further study of wear calculation and corrugation.
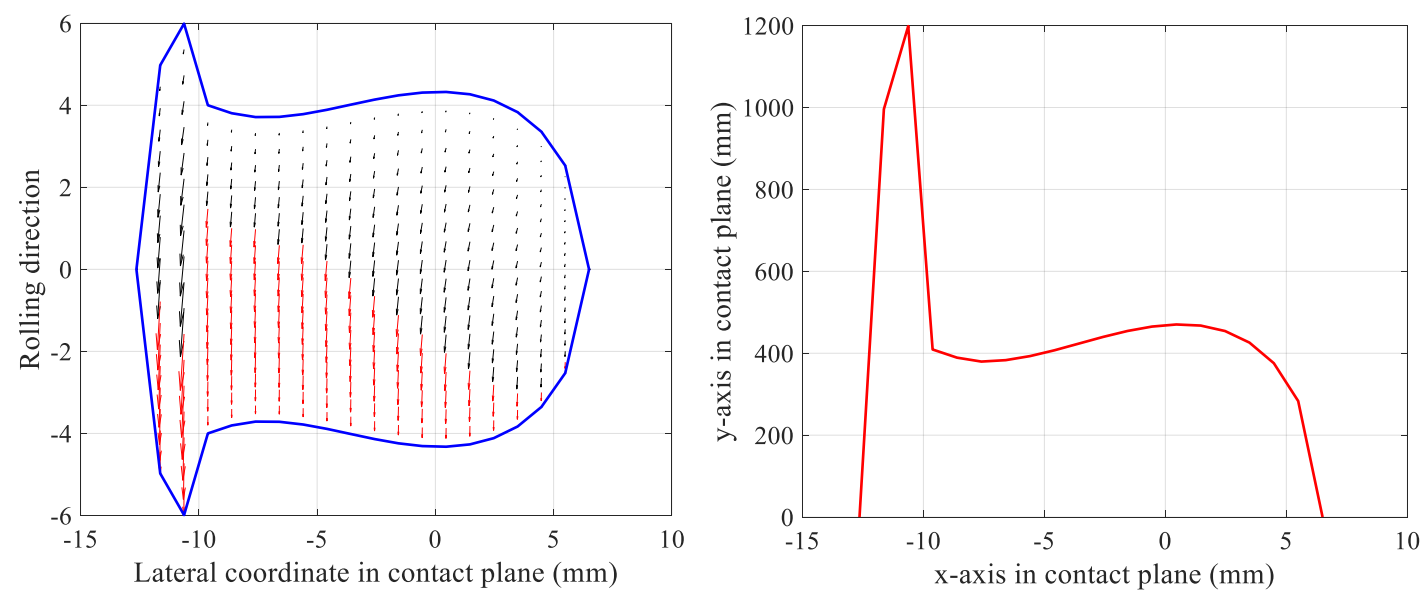

Figure 8. Normal and tangential solution with the contact patch divided in 19 strips (60 kN normal load, $0.1 \%$ longitudinal and lateral creepage).

\section{Results and discussion}

In this section, some numerical results of train-track interaction numerical simulation are presented and analyzed for one case considering sinusoidal rail corrugation and for one case considering random rail corrugation. The simulation considers the vehicle running at $300 \mathrm{~km} / \mathrm{h}$ along a tangent track with a wheel load of $60 \mathrm{kN}$. Simulation 
parameters related to the wheelset and the track can be found in Table 1. Simulation results are compared in terms of wheel/rail contact forces and vibration spectra evaluated at different points on the rail section. Firstly, the wheel/rail contact forces under sinusoidal rail corrugation for different model options are investigated. Secondly, the wheel/rail contact forces and vibration spectra under a random rail corrugation are investigated to study the effect of rail flexibility with a focus on the high frequency range, i.e. for frequencies above $1 \mathrm{kHz}$.

Table 1 Simulation parameters

\begin{tabular}{c|c}
\hline Description & Value \\
\hline Wheelset type & S1002 \\
\hline Wheelset mass & $1375 \mathrm{~kg}$ \\
\hline Axle load & $120 \mathrm{kN}$ \\
\hline Rail type & UIC 60 \\
\hline Sleeper spacing & $0.6 \mathrm{~m}$ \\
\hline Track length & $48 \mathrm{~m}$ \\
\hline Rail support vertical stiffness & $3 \times 10^{8} \mathrm{~N} / \mathrm{m}$ \\
\hline Rail support vertical damping & $3 \times 10^{4} \mathrm{Ns} / \mathrm{m}$ \\
\hline Rail support lateral stiffness & $2 \times 10^{7} \mathrm{~N} / \mathrm{m}$ \\
\hline Rail support lateral damping & $8 \times 10^{3} \mathrm{Ns} / \mathrm{m}$ \\
\hline Rail support longitudinal stiffness & $2 \times 10^{7} \mathrm{~N} / \mathrm{m}$ \\
\hline Rail support longitudinal damping & $8 \times 10^{3} \mathrm{Ns} / \mathrm{m}$ \\
\hline Ballast vertical stiffness & $8 \times 10^{7} \mathrm{~N} / \mathrm{m}$ \\
\hline Ballast vertical damping & $1 \times 10^{5} \mathrm{Ns} / \mathrm{m}$ \\
\hline Ballast lateral stiffness & $8 \times 10^{6} \mathrm{~N} / \mathrm{m}$ \\
\hline Ballast lateral damping & $8 \times 10^{3} \mathrm{Ns} / \mathrm{m}$ \\
\hline Ballast longitudinal stiffness & $8 \times 10^{6} \mathrm{~N} / \mathrm{m}$ \\
\hline Ballast longitudinal damping & $8 \times 10^{3} \mathrm{Ns} / \mathrm{m}$ \\
\hline
\end{tabular}

\subsection{Responses to sinusoidal rail corrugation}

In this section, two harmonic profiles of rail corrugation with different wavelengths are selected to see the influence of wheelset, track and contact options on the contact forces. The amplitude of the corrugation is set to be $48.6 \mu \mathrm{m}$, which is the same as one case reported in [3].

\subsubsection{Wheel-rail contact force for different track options}

In order to study the effect of rail flexibility on wheel-rail contact forces, a sinusoidal rail corrugation with $0.06 \mathrm{~m}$ wavelength is selected, which is one tenth of the sleeper bay. With the vehicle velocity being $300 \mathrm{~km} / \mathrm{h}$, the excitation frequency in this case is $1389 \mathrm{~Hz}$. Three different options for track modelling are considered, in combination 
with a rotating flexible wheelset and a non-Hertzian/FASTSIM contact model. The three track models considered are: a Timoshenko beam element discrete-supported track model, a 3D ALE continuous-supported track model and a 3D ALE discretesupported track model are considered in this study. The Timoshenko beam track model comes from the authors' previous work [32]. This track model considers both the vertical and the lateral movement of Timoshenko beam element and is proved to be valid in the frequency range up to $1.5 \mathrm{kHz}$ in vertical direction and $1 \mathrm{kHz}$ in lateral direction. The result from the Timoshenko model is firstly compared with the output from an established model developed in literature [3], which contains a flexible wheelset, a modal-based Timoshenko-beam track and a Hertzian/FASTSIM contact model. Moreover, the responses with 3D discrete-supported track model and 3D continuous-supported track model are compared with a Timoshenko beam track model from the authors' previous work [32].

Fig. 9 compares the time histories of the vertical contact forces with different model configurations. In Fig. 9(a) this quantity is compared for two simulations performed using the Timoshenko beam track model in combination with the non-Hertzian EKP contact model and a Hertzian contact model, respectively. The two results show nearly the same amplitude and trend, indicating that the effect of using a non-Hertzian contact model is quite weak as far as the calculation of the normal contact force is considered. Fig. 9(b) shows the vertical contact force using the three different track models: Timoshenko beam on discrete supports, 3D solid FE on continuous support and 3D solid FE on discrete supports. Both models considering the discrete support of the rail give rise to two harmonic components of the normal contact force, one corresponding to the exciting corrugation wavelength and the other caused by the sleeper passing effect whose wavelength is determined by the sleeper bay $(0.6 \mathrm{~m})$. In the case of the continuously-supported rail model, the sleeper passing effect is not accounted for by the model and, as a consequence, the dynamic fluctuation of the contact force is significantly reduced compared to the other two cases. The model based on Timoshenko beams on discrete supports shows a slightly higher force fluctuation compared to the solid FE model with discrete track support. This is due to the fact that the beam model of the track has a slightly higher mechanical impedance in the high frequency range, as it inherently introduce a fictitious constraint by assuming a rigid motion of the rail sections. 


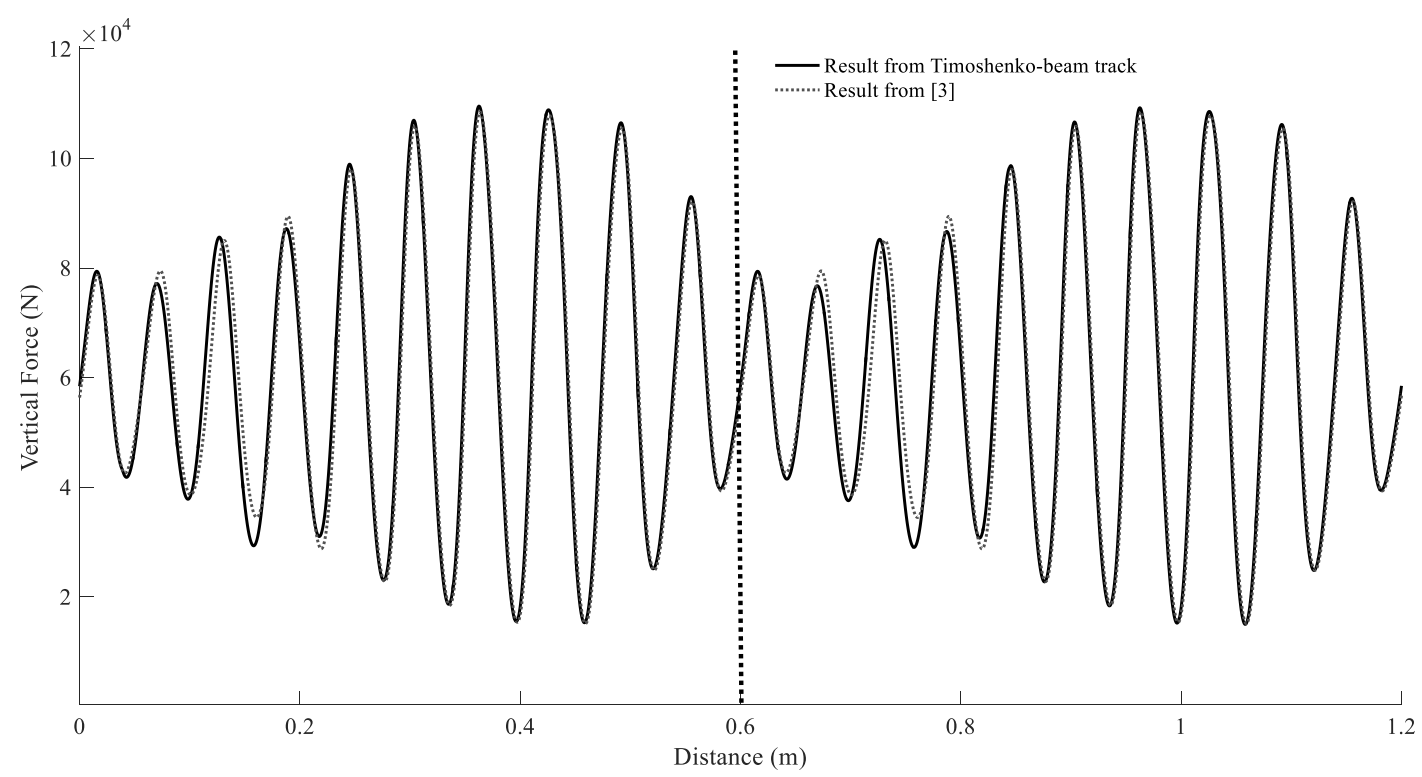

(a)

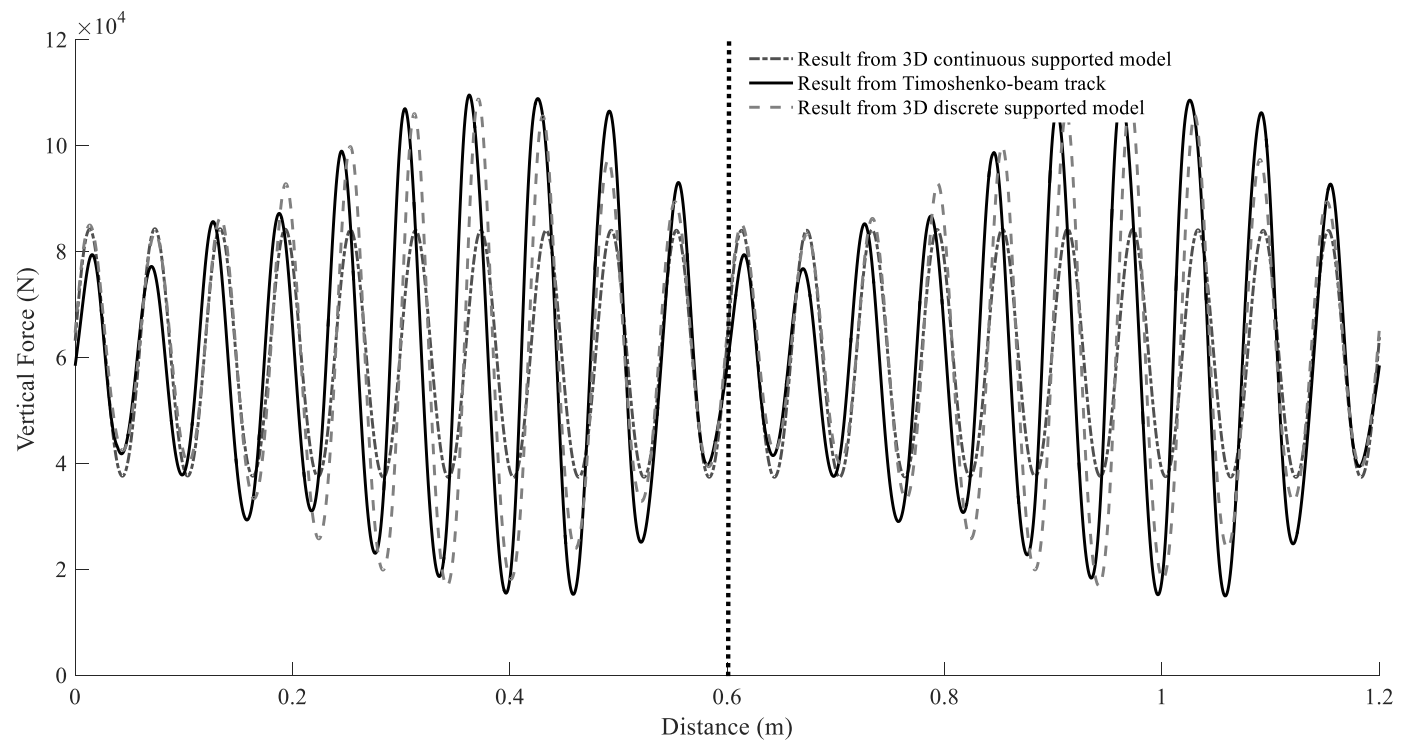

(b)

Figure 9. Wheel-rail vertical contact force when the wheelset travels on a corrugated track with different model configurations (the dotted vertical line indicates position of the rail support).

Fig. 10 shows the lateral contact forces for different track models. Similar to the analysis of vertical force in Fig. 9, Fig. 10(a) compares the lateral contact force for the Timoshenko beam track model with the non-Hertzian EKP contact model and with the Hertzian contact model respectively. In this case, the results obtained using the two different contact models shows some non-negligible difference, the dynamic fluctuations calculated using the non-Hertzian model being generally larger than for the Hertzian model. Fig. 10(b) shows the lateral contact force using the three different track models. For this contact force component the 3D solid FE model with continuous rail 
support predicts a lower fluctuation of the force as it does not consider the excitation caused by the sleeper passing effect. Furthermore, the 3D solid FE model with discretely supported rail shows a larger fluctuation of the lateral force compared to the Timoshenko beam model, since the Timoshenko beam element fails to describe lateral cross section flexibility of the rail which becomes important at frequencies higher than $1 \mathrm{kHz}$.

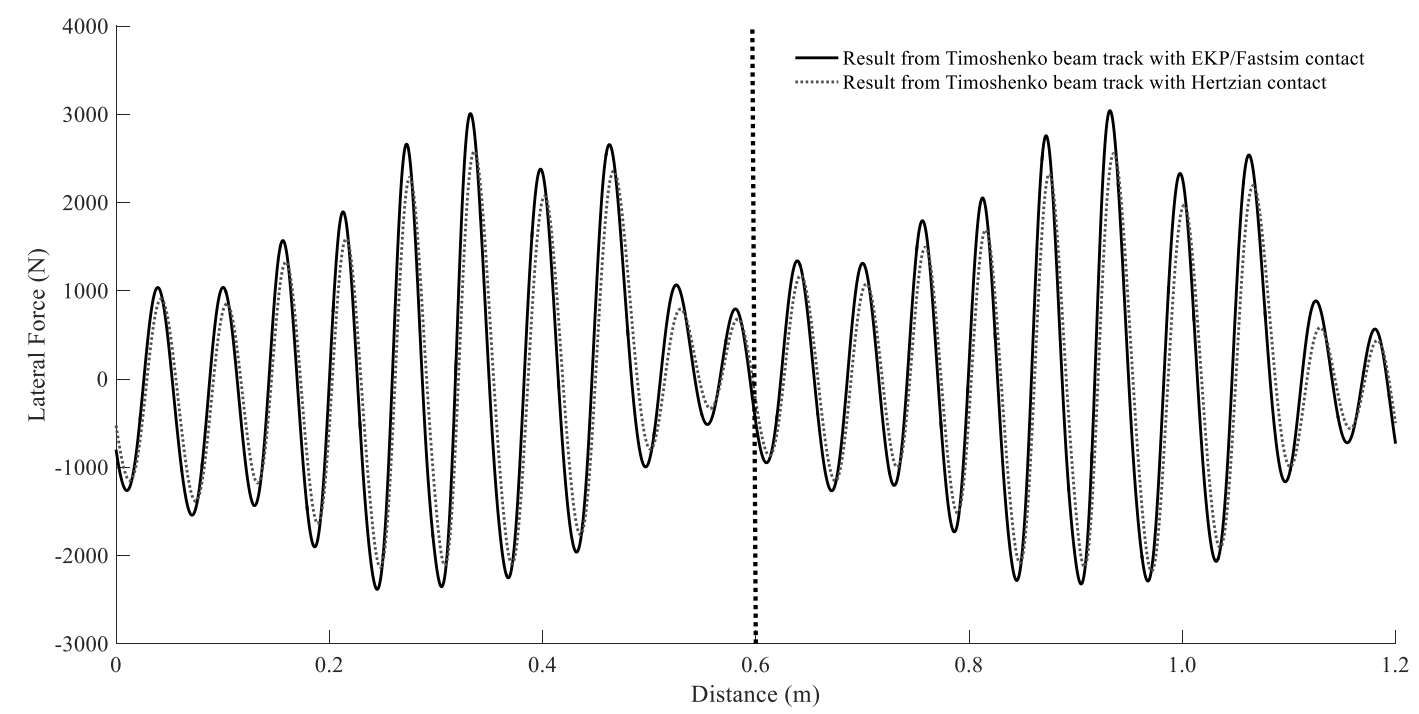

(a)

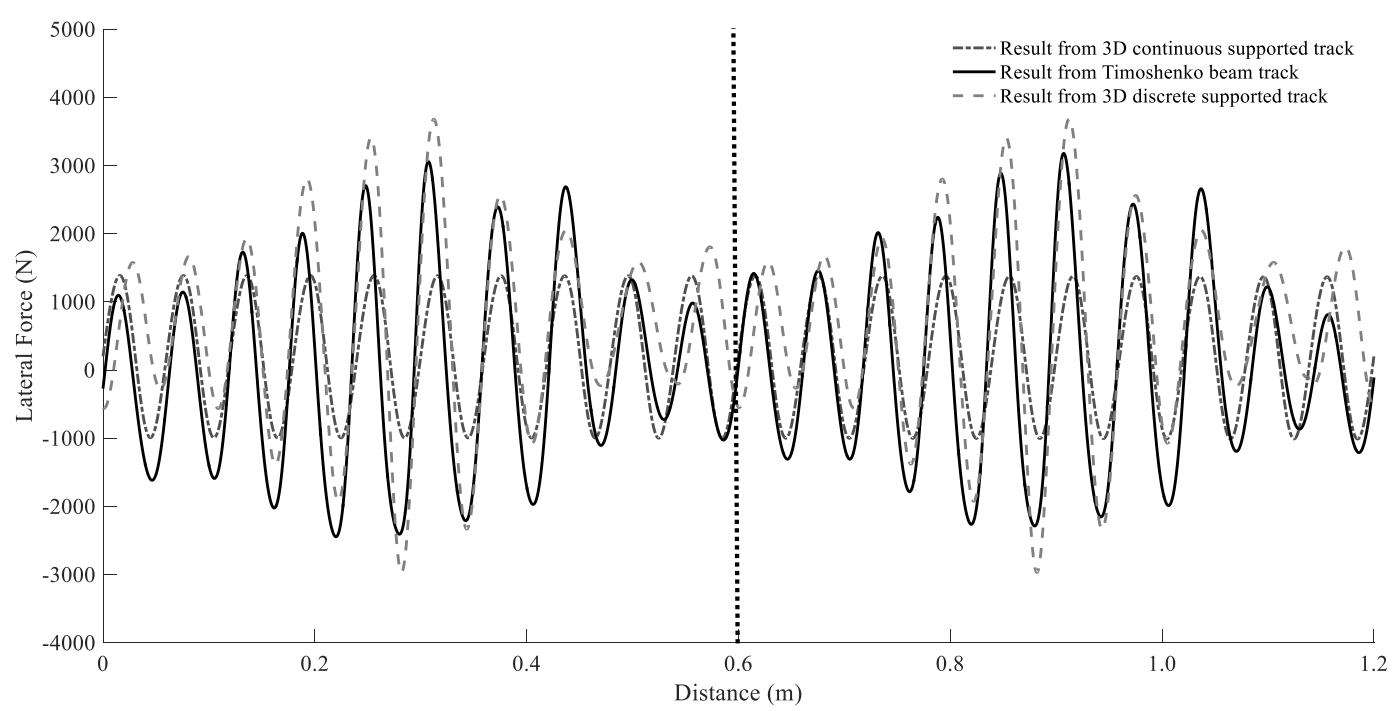

(b)

Figure 10. Wheel-rail lateral contact force when the wheelset travels on a corrugated track with different model configurations (the dotted vertical line indicates position of the rail support). 


\subsubsection{Wheel-rail contact force for different wheelset options}

In order to study the effect of wheelset flexibility on wheel-rail contact forces, a sinusoidal rail corrugation with $0.053 \mathrm{~m}$ wavelength is selected. With a vehicle speed of $300 \mathrm{~km} / \mathrm{h}$, the corresponding frequencies of the corrugation is $1570 \mathrm{~Hz}$, which is related to the $4^{\text {th }}$ backward wheelset bending mode of the rotating wheelset.

Together with a non-Hertzian/FASTSIM contact model and a 3D ALE discretesupported track model, a rigid wheelset model and a 3D ALE flexible wheelset model are considered in this study. Fig. 11 compares the vertical and lateral contact forces for the different wheelset models. Considering first the vertical forces shown in Fig. 11(a), the results obtained considering the rigid wheelset and flexible wheelset models are similar, leading to the conclusion that the influence of wheelset flexibility is negligible in the vertical direction. For the lateral direction in Fig. 11(b), more clear difference can be found between the rigid wheelset and the rotating flexible wheelset. The maximum amplitude obtained considering the flexible wheelset model is nearly 2.5 times higher than the same result when a rigid wheelset model is used, indicating a remarkable influence of wheelset flexibility.

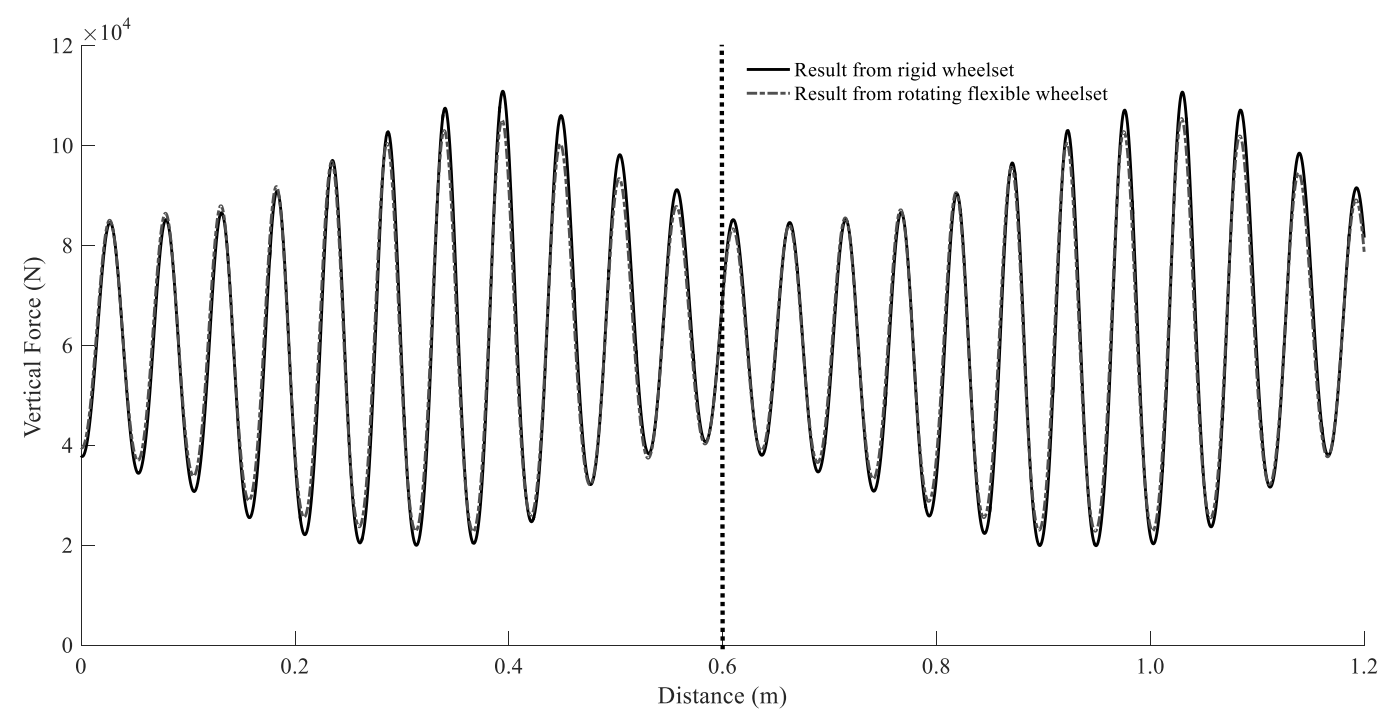

(a) 


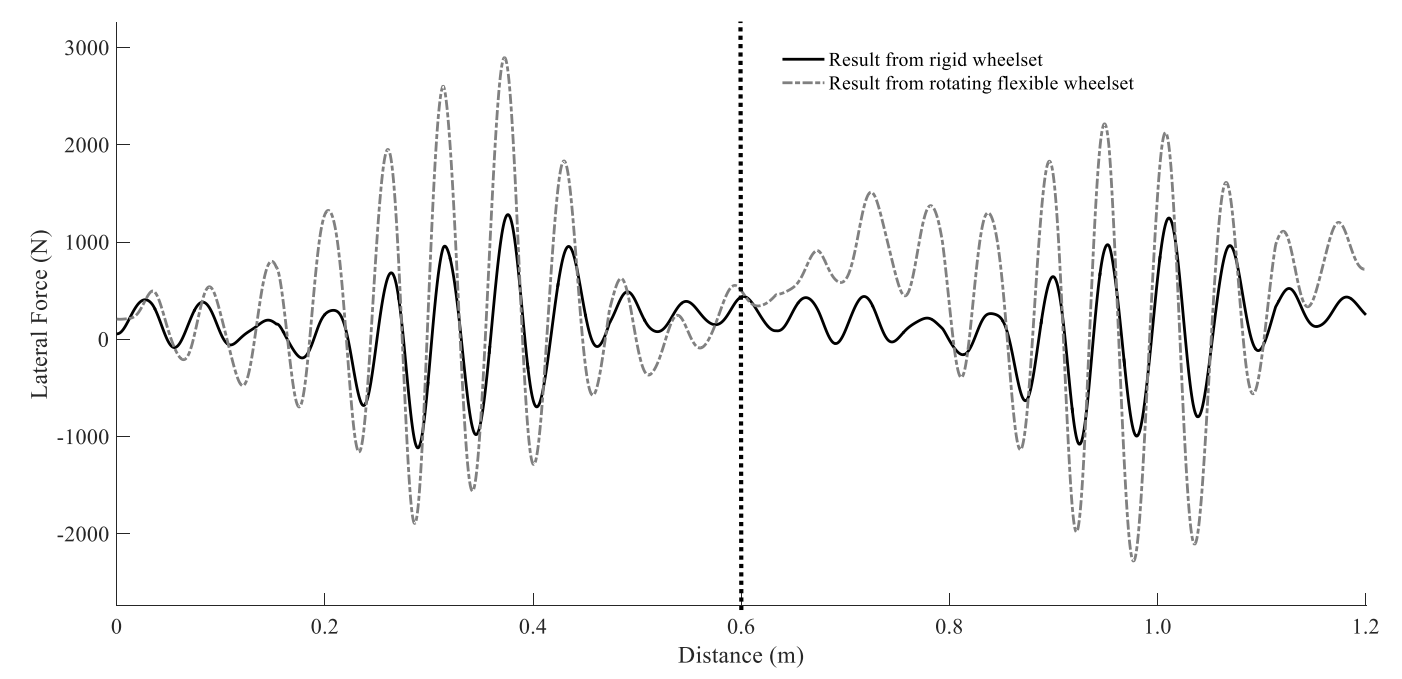

(b)

Figure 11. Contact forces with a harmonic exciting frequency of $1570 \mathrm{~Hz}$ : (a) vertical contact force; (b) lateral contact force.

\subsection{Responses to random rail corrugation}

In this section, the wheelset-track interaction dynamic responses in the high frequency range are examined considering excitation coming from a random rail corrugation. The corrugation profile considered is a space realization of a $1 / 3$ octave band spectrum defined by ISO 3095:2005 standard [40], covering a range of wavelengths from $0.01 \mathrm{~m}$ to $0.63 \mathrm{~m}$. The vehicle velocity is $300 \mathrm{~km} / \mathrm{h}$, leading to a frequency range from $132 \mathrm{~Hz}$ to $8 \mathrm{kHz}$ for the excitation.

\subsubsection{Analysis of wheel-rail contact force}

Fig. 12(a) shows the dynamic contact force in vertical direction for a 0.2 second simulation, and in Fig. 12(b) a detail of the same time history, to better appreciate the differences between the three track models considered. The dynamic fluctuation of the force is much larger than for the case of harmonic excitation considered in Fig. 8. Therefore, the influence of the sleeper passing frequency is in this case not clearly visible, as the effect of rail roughness is prevailing. The Timoshenko beam model shows a similar fluctuation trend compared to the 3D solid FE model with discretely supported rail, while the 3D solid FE model with continuously supported rail shows larger difference compared to the other two cases.

For the lateral force, see Fig. 13, larger differences are observed in the results obtained using the three models of the track. The results of the two models considering the discrete support of the track show for this component of the contact force the typical wavelength corresponding to the sleeper bay which is due to the sleeper-passing 
excitation.
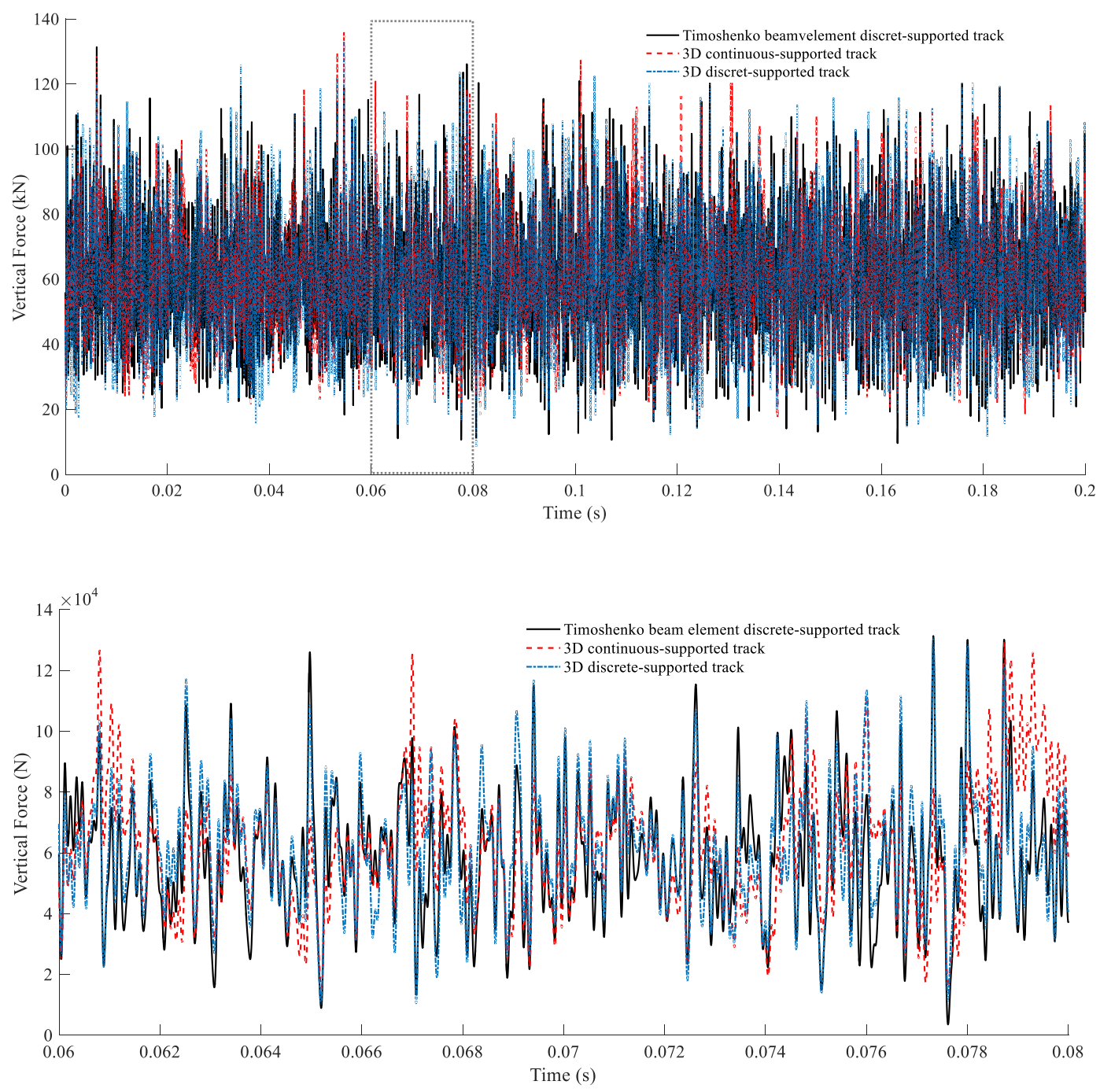

Figure 12. Vertical contact forces of the 3D wheelset-track interaction model for different contact models and excitation caused by random track irregularities. a) entire time history $(0.2 \mathrm{~s}) \mathrm{b})$ detail $(0.02 \mathrm{~s})$.

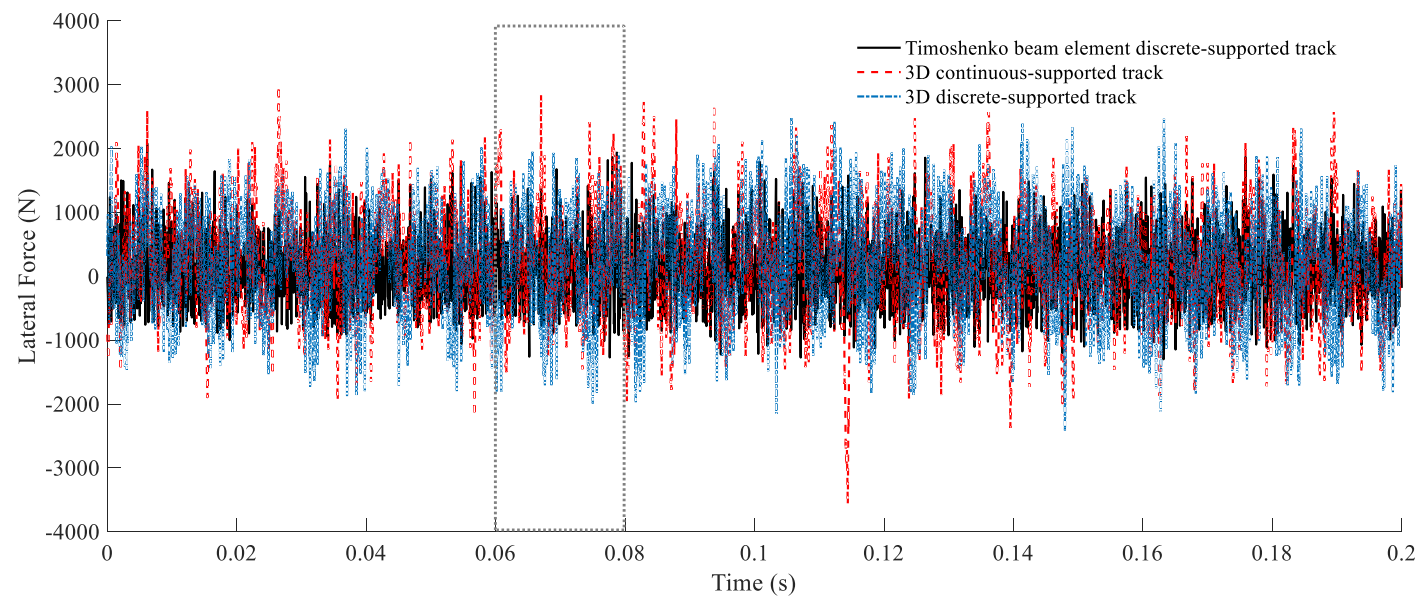




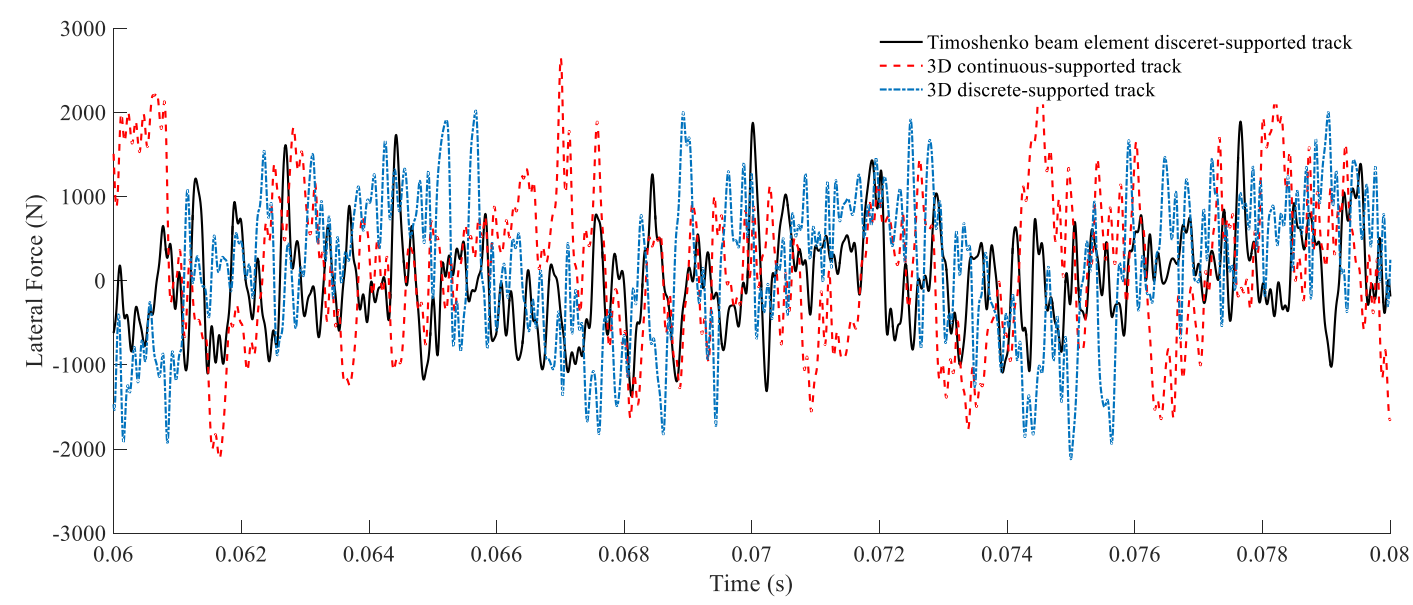

Figure 13. Lateral contact forces of the 3D wheelset-track interaction model for different track options and excitation caused by random track irregularities. a) entire time history ( $0.2 \mathrm{~s})$ b) detail $(0.02 \mathrm{~s})$.

For further analysis, the $1 / 3$ octave band spectra of the vertical and lateral contact forces for different track option are investigated, see Fig. 14. In both diagrams, a peak is observed at about $140 \mathrm{~Hz}$, corresponding to the sleeper passing frequency, for the two models with discrete rail support. However, the sleeper passing influence is much more clearly visible for the lateral component of the contact force, which is consistent with the analysis of the signals in the time domain. The spectra of the vertical force for the two models with discrete rail support also show another peak at the pinned-pinned frequency, close to $1 \mathrm{kHz}$. The spectrum of the vertical contact force obtained using the Timoshenko beam model is quite similar to the one obtained using the $3 \mathrm{D}$ solid FE model with discretely supported rail up to $2 \mathrm{kHz}$ approximately, but fails to describe the harmonic components of rail vibration for higher frequencies due to the fact that the Timoshenko beam element cannot reproduce the dynamics of the rail associated with non-rigid motion of the rail section. In the lateral direction, the Timoshenko beam model shows a significantly lower force component at frequencies higher than $1 \mathrm{kHz}$, compared to both continuously and discretely supported 3D solid FE models. On the other hand, the 3D solid FE model with continuously supported rail fails to describe several resonances caused by the discrete support up to a mid-high frequency. However, in a frequency range higher than $2 \mathrm{kHz}$ this model shows a good agreement with the 3D solid FE model with discretely supported rail. 

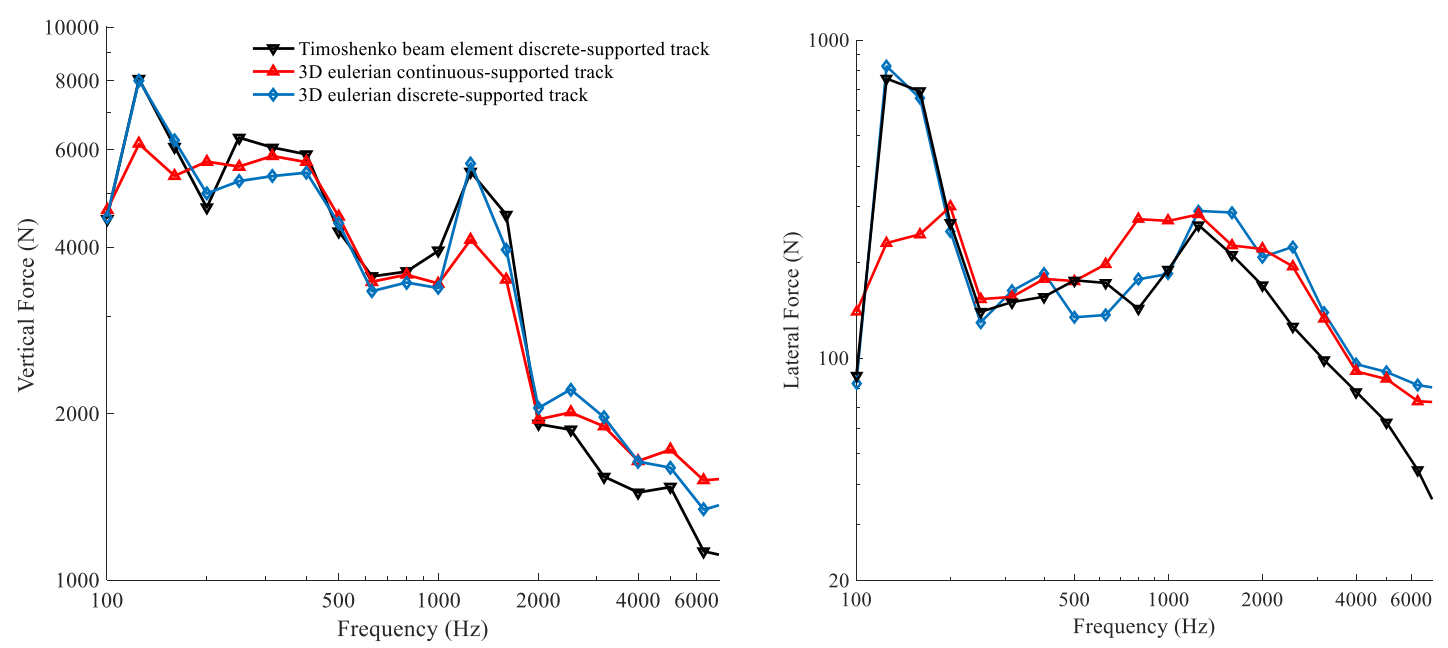

Figure 14. 1/3 Octave spectrum of contact forces of the 3D wheelset-track interaction model for different track options and excitation caused by random track irregularities. Left: vertical force. Right: lateral force.

\subsubsection{Analysis of rail vibration}

In this section, the normal component of the rail vibration velocity is considered for three different positions in the rail section, namely at the railhead, rail web and rail foot, points 1, 2 and 3 respectively in Fig. 15. These quantities are directly related to noise emission from different portions of the rail surface.

For the two solid FE models, the normal velocity at each point is evaluated as the projection along the normal direction of the vertical and lateral components of the velocity vector at one single node of the FE model, whereas for the Timoshenko beam model the normal velocity at the three considered locations is evaluated assuming a rigid motion of the rail section. 


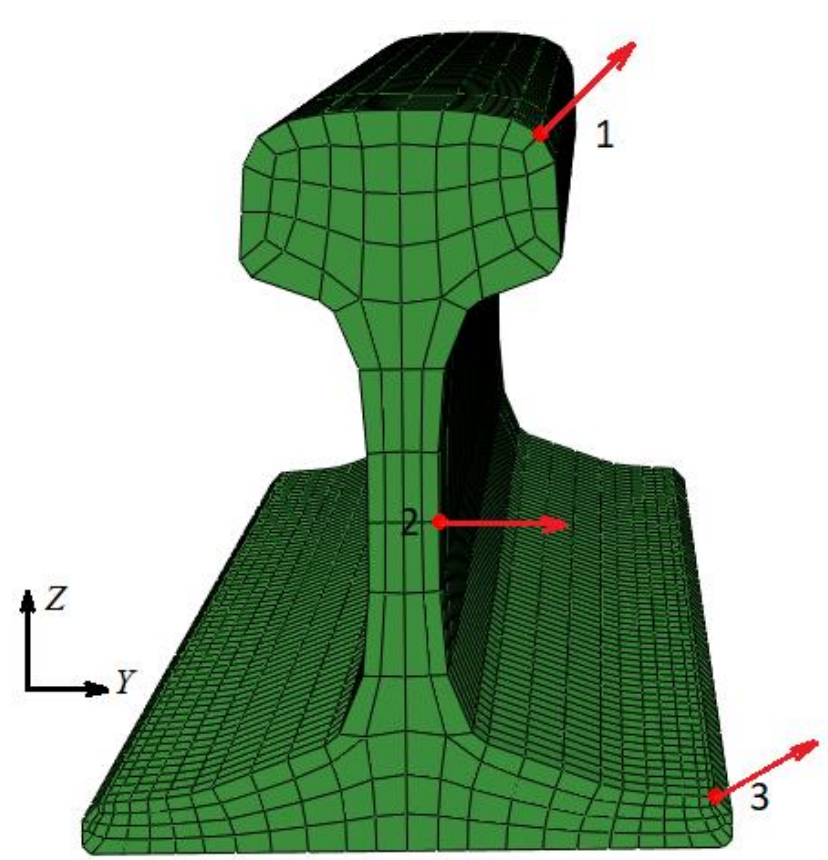

Figure 15. Finite element mesh of the rail section and position of points 1, 2, 3 at which the normal velocity of the rail is evaluated.

Fig. 16 to 18 show the $1 / 3$ octave band spectrum of the normal velocity for the three track models considered. In a frequency range up to $1 \mathrm{kHz}$, the spectra obtained using the Timoshenko beam model and the solid FE model with discrete rail support are very similar, confirming that the Timoshenko beam model reproduces correctly the vibration of the rail in this frequency range. However, at higher frequencies, the results of the two models show remarkable differences at all positions, with the solid FE model predicting larger vibration velocity by +2.5 to $4.5 \mathrm{~dB}$ on average. On the other hand, the 3D solid FE model with continuously supported rail shows significant deviations from the same model but with discretely supported rail in a frequency range up to 1.5 $\mathrm{kHz}$. These difference are mostly due to the fact that the model with continuous rail support does not consider the excitation deriving from the sleeper passing at $140 \mathrm{~Hz}$ and some track resonances e.g. the P2 resonance at about $400 \mathrm{~Hz}$, the pinned-pinned resonance in vertical and lateral direction at about $1 \mathrm{kHz}$. However, at higher frequencies, the vibration response of the two solid FE models shows a good consistency with each other. 


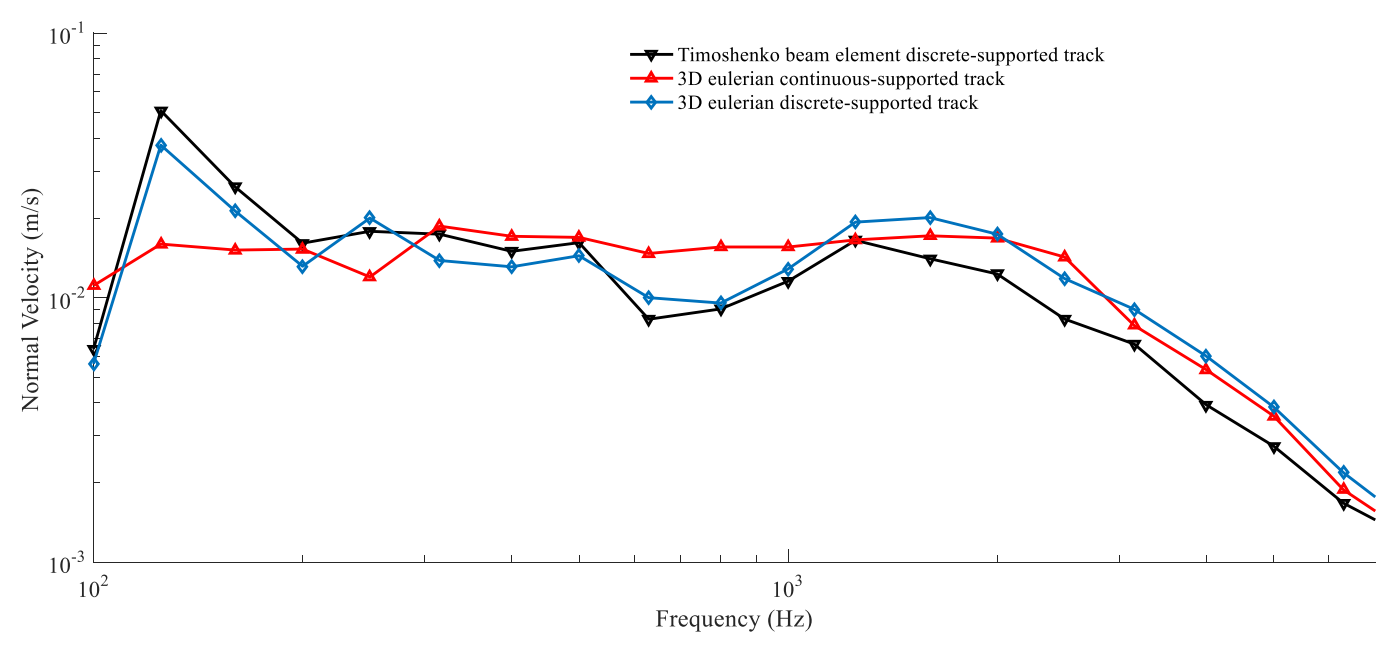

Figure 16. 1/3 Octave spectrum of normal velocity at location 1 in Fig. 12 for different track options.

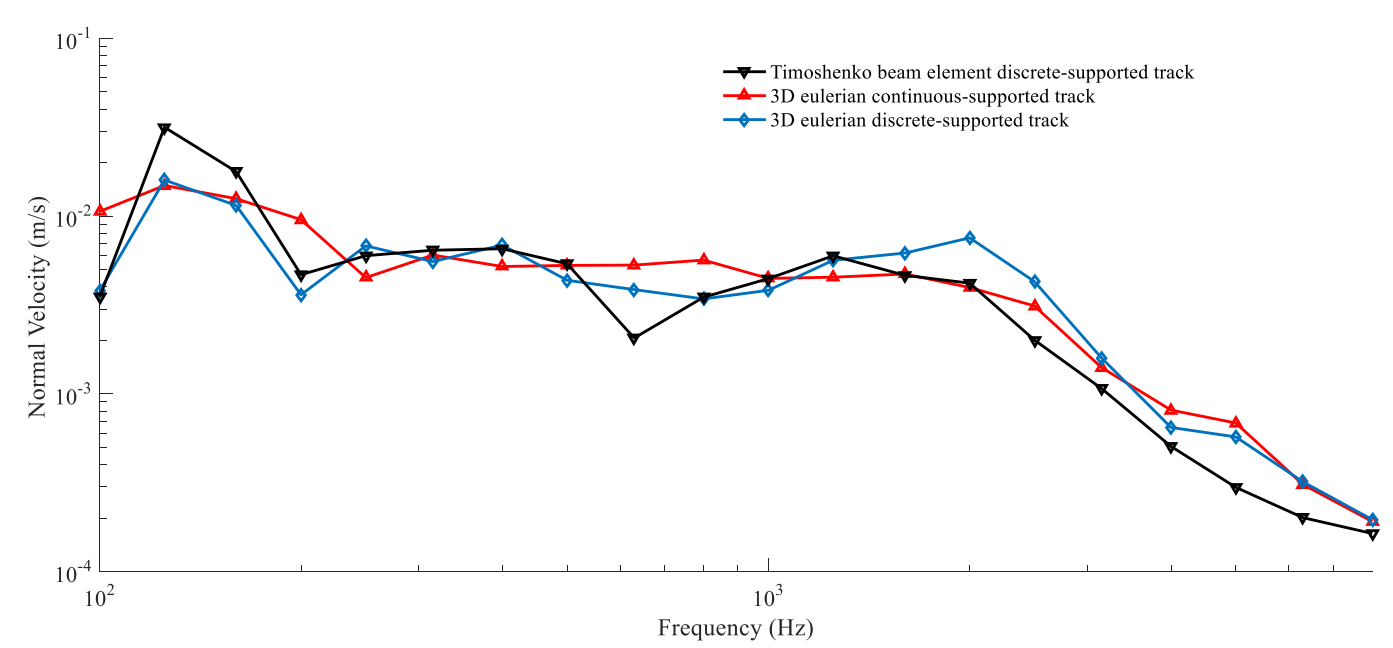

Figure 17. 1/3 Octave spectrum of normal velocity at location 2 in Fig. 12 for different track options.

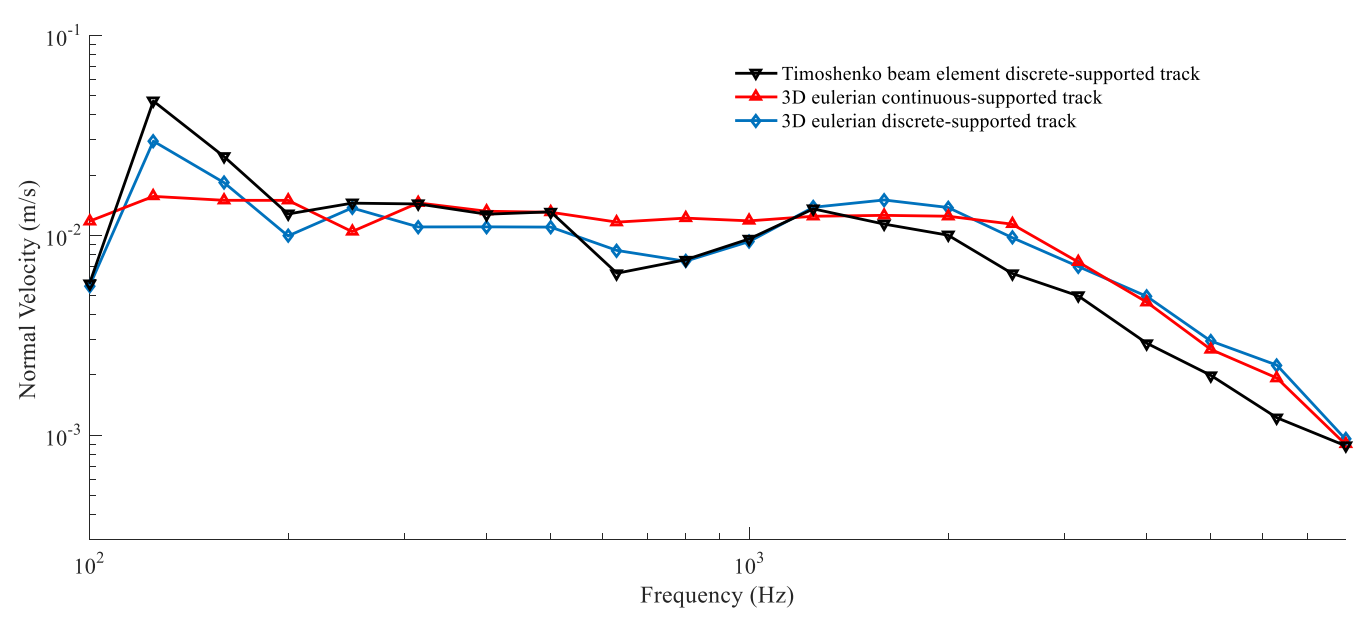

Figure 18. 1/3 Octave spectrum of normal velocity at location 3 in Fig. 12 for different track options. 
Table 2 reports an estimate of the computational effort required by the calculation for different wheelset models and track models in presence of random rail corrugation. The table shows that the flexible wheelset model combined with the 3D ALE model of the rails on discrete supports requires the largest computational effort. discrete supported track. Considering the same wheelset model and the continuously supported 3D ALE model of the rails results in approximately reducing to $50 \%$ the CPU time, since the calculation of the time-varying forces generated by the discrete supports is no longer required. Using the Timoshenko beam model of the track results in a CPU time required which is similar to the case of the continuously supported 3D rail model. Finally, using a rigid model of the wheelset results in further reduction of the CPU time compared to considering wheelset flexibility, due to the lower number of modal coordinates involved in the simulation. Therefore, the choice of the most appropriate model for wheelset and track flexibility should be the result of a best deal between accuracy and computational effort, which normally depends on the problem under study.

Table 2 CPU time (considering an Intel(R) Core(TM) i7-6700HQ CPU 2.60GHZ processor)

required for performing a $0.2 \mathrm{~s}$ numerical simulation in presence of random rail corrugation

\begin{tabular}{c|c}
\hline Model options & CPU time (min) \\
\hline Rigid wheelset + Timoshenko beam track & 11 \\
\hline Flexible wheelset + Timoshenko beam track & 23 \\
\hline Rigid wheelset + 3D continuous supported track & 10 \\
\hline Flexible wheelset +3 D continuous supported track & 21 \\
\hline Rigid wheelset +3 D discrete supported track & 31 \\
\hline Flexible wheelset + 3D discrete supported track & 43 \\
\hline
\end{tabular}

\section{Conclusion}

This paper proposed a novel time domain rotating flexible wheelset-3D track interaction model in an ALE approach together with a non-Hertzian EKP contact, which is valid for dynamic simulation up to $7 \mathrm{kHz}$. This work helps to extend the valid frequency range of current train-track interaction models with limited time effort by introducing an ALE coordinate to the FE wheelset and track model. Moreover, by introducing the non-Hertzian non-linear EKP wheel/rail contact, the interaction model can be used to investigate the formation of short pitch corrugation on the rail head and on the wheel tread. The effect of different track options on wheel/rail contact forces and rail section vibrations was also investigated.

Based on the comparison of contact forces and rail vibrations using three different track options, the performance of different wheelset-track interaction models was 
discussed. On the one hand, neglecting discrete support of the rail may lead to an underestimation of the dynamic contact forces generated by rail support resonances below $1.5 \mathrm{kHz}$, on the other hand, using a simplified Timoshenko beam track model for track dynamics may have a serious impact on contact forces and rail vibration for frequencies higher than 1-1.5 kHz. Moreover, the Timoshenko beam element fails to describe the deformation of the rail section, which has a remarkable influence on rail web and rail foot vibration. Overall, the results show that simulation results are highly sensitive to the track model adopted. Considering the computational cost, the Timoshenko beam track model can be used for interaction problems below $1 \mathrm{kHz}$, such as studying the effect of wheel flats and wheel out-of-roundness, while the 3D solid FE model with continuously supported rail may be applied in wheel-rail interaction problems concentrating on the high frequency range, such as high frequency rail-wheel noise generation.

The 3D ALE wheelset-discrete-supported track interaction model in this paper can be used for investigating specific interaction problems in the entire frequency range from tens of $\mathrm{Hz}$ to some $\mathrm{kHz}$, at the expense of a higher computational effort required, compared to the other two models. Future developments of this work will be addressed to using the train-track interaction models developed in the study of railhead corrugation and rolling noise.

Acknowledgements: The first author of this work has been financially supported by China Scholarship Council (Grant No. 201407090055).

\section{References}

1. D, Thompson.: Railway noise and vibration: mechanisms, modelling and means of control. Elsevier (2008).

2. W, Sun., et al.: Vertical random vibration analysis of vehicle-track coupled system using Green's function method. Vehicle System Dynamics. 52: 362-389 (2014)

3. J, Martínez-Casas., et al.: Numerical estimation of stresses in railway axles using a traintrack interaction model. International Journal of Fatigue. 47: 18-30 (2013)

4. J.C., Nielsen. and A, Johansson.: Out-of-round railway wheels-a literature survey. Proceedings of the Institution of Mechanical Engineers, Part F: Journal of Rail and Rapid Transit. 214: 79-91 (2000)

5. J.C., Nielsen., A, Ekberg. and R, Lundén.: Influence of short-pitch wheel/rail corrugation on rolling contact fatigue of railway wheels. Proceedings of the Institution of Mechanical Engineers, Part F: Journal of Rail and Rapid Transit. 219: 177-187 (2005)

6. D, Thompson. and C, Jones.: A review of the modelling of wheel/rail noise generation. Journal of sound and vibration. 231: 519-536 (2000)

7. A.A., Shabana., K.E. Zaazaa. and H, Sugiyama.: Railroad vehicle dynamics: a computational approach. CRC press (2007)

8. L, Baeza., et al.: High frequency railway vehicle-track dynamics through flexible rotating 
wheelsets. Vehicle System Dynamics. 46: 647-659 (2008)

9. L, Baeza., et al.: Prediction of rail corrugation using a rotating flexible wheelset coupled with a flexible track model and a non-Hertzian/non-steady contact model. Journal of Sound and Vibration. 330: 4493-4507 (2011)

10. J, Arnold., I, Kaiser. and G, Schupp.: Simulation of a railway vehicle's running behaviour: how elastic wheelsets influence the simulation results. Vehicle System Dynamics. 41: 242251 (2005)

11. I, Kaiser. and K, Popp.: Interaction of elastic wheelsets and elastic rails: modelling and simulation. Vehicle System Dynamics. 44: 932-939 (2006)

12. I, Kaiser.: Refining the modelling of vehicle-track interaction. Vehicle System Dynamics. 50: 229-243 (2012)

13. I, Kaiser., et al.: The impact of structural flexibilities of wheelsets and rails on the hunting behaviour of a railway vehicle. Vehicle system dynamics. DOI: 10.1080/00423114.2018.1484933 (2018)

14. A.A., Shabana.: Dynamics of multibody systems. Cambridge university press (2013)

15. M, Brown. and A.A., Shabana.: Application of multibody methodology to rotating shaft problems. Journal of Sound and Vibration. 204: 439-458 (1997)

16. E, Di Gialleonardo., F, Braghin. and S, Bruni.: The influence of track modelling options on the simulation of rail vehicle dynamics. Journal of Sound and Vibration. 331: 4246-4258 (2012)

17. L, Baeza. and H, Ouyang.: A railway track dynamics model based on modal substructuring and a cyclic boundary condition. Journal of Sound and Vibration. 330: 75-86 (2011)

18. K, Knothe. and S, Grassie.: Modelling of railway track and vehicle/track interaction at high frequencies. Vehicle system dynamics. 22: 209-262 (1993)

19. R, Chamorro., J.L., Escalona. and M, González.: An approach for modeling long flexible bodies with application to railroad dynamics, Multibody System Dynamics. 26: 135-152 (2011)

20. F, Ripamonti., R, Ariente. and S, Bruni.: A time domain model for the study of high frequency train-track interaction. $7^{\text {th }}$ International Conference on Railway Bogies and Running Gears. Budapest, Hungary (2007)

21. J, Martínez-Casas., et al.: Improved railway wheelset-track interaction model in the highfrequency domain. Journal of Computational and Applied Mathematics. 309: 642-653 (2017)

22. C, Koh., G, Chiew. and C, Lim.: A numerical method for moving load on continuum. Journal of sound and vibration. 300: 126-138 (2007)

23. H, Hertz.: Über die Berührung fester elastischer Körper. (1882)

24. W, Kik. and J, Piotrowski.: A fast, approximate method to calculate normal load at contact between wheel and rail and creep forces during rolling. $2^{\text {nd }}$ Mini Conference on Contact Mechanics and Wear of Rail/Wheel System, TU Budapest, Hungary (1996)

25. J, Piotrowski. and H, Chollet.: Wheel-rail contact models for vehicle system dynamics including multi-point contact. Vehicle System Dynamics. 43: 455-483 (2005)

26. J, Piotrowski. and W, Kik.: A simplified model of wheel/rail contact mechanics for nonHertzian problems and its application in rail vehicle dynamic simulations. Vehicle System Dynamics. 46: 27-48 (2008)

27. J, Kalker.: Wheel-rail rolling contact theory. Wear. 144: 243-261 (1991) 
28. J, Ayasse. and $\mathrm{H}$, Chollet.: Determination of the wheel rail contact patch in semi-Hertzian conditions. Vehicle System Dynamics. 43: 161-172 (2005)

29. T, Telliskivi. and U, Olofsson.: Contact mechanics analysis of measured wheel-rail profiles using the finite element method. Proceedings of the Institution of Mechanical Engineers, Part F: Journal of Rail and Rapid Transit. 215: 65-72 (2001)

30. X, Zhao. and Z, Li.: The solution of frictional wheel-rail rolling contact with a 3D transient finite element model: Validation and error analysis. Wear. 271: 444-452 (2011)

31. B, Liu., S, Bruni. and E, Vollebregt.: A non-Hertzian method for solving wheel-rail normal contact problem taking into account the effect of yaw. Vehicle System Dynamics. 54: 12261246 (2016)

32. W, Fang., J, Martinez-Casas. and S, Bruni.: A Time-Domain Model for the Study of HighFrequency Wheelset-Track Interaction. Urban Rail Transit. 3: 203-213 (2017)

33. H, Irschik., H.J., Holl.: The equations of Lagrange written for a non-material volume. ActaMech. 153: 231-248 (2002)

34. K, Knothe. and A, Groß-Thebing.: Short wavelength rail corrugation and non-steady-state contact mechanics. Vehicle system dynamics. 46: 49-66 (2008)

35. X, Jin. and Z, Wen.: Effect of discrete track support by sleepers on rail corrugation at a curved track. Journal of sound and Vibration. 315: 279-300 (2008)

36. G, Diana., et al.: Experimental and numerical investigation on subway short pitch corrugation. Vehicle System Dynamics. 29: 234-245 (1998)

37. F, Braghin., S, Bruni. and G, Diana.: Experimental and numerical investigation on the derailment of a railway wheelset with solid axle. Vehicle System Dynamics. 44: 305-325 (2006)

38. J, Kalker.: A fast algorithm for the simplified theory of rolling contact. Vehicle system dynamics. 11: 1-13 (1982)

39. F, Braghin., S, Bruni. and R, Lewis.: Railway wheel wear. In: Lewis, R and Olofsson, Wheel/rail interface handbook. 1-59. Woodhead Publishing Ltd (2009)

40. ISO, E., 3095.: Railway applications-acoustics-measurement of noise emitted by railbound vehicles (2005) 